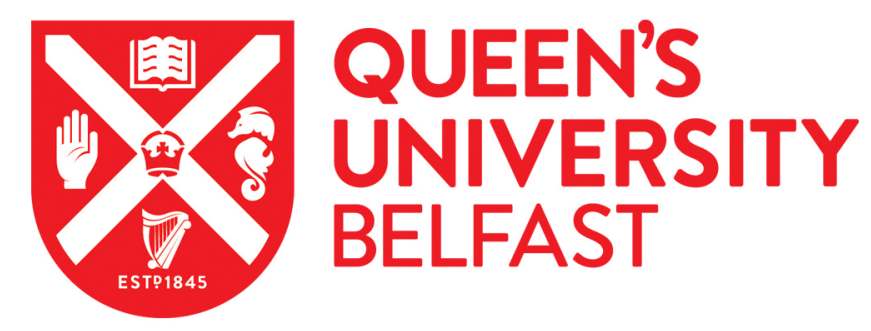

\title{
Proactive Relay Selection with Joint Impact of Hardware Impairment and Co-channel Interference
}

Duy, T. T., Duong, T. Q., da Costa, D. B., Bao, V. N. Q., \& Elkashlan, M. (2015). Proactive Relay Selection with Joint Impact of Hardware Impairment and Co-channel Interference. IEEE Transactions on Communications, 63(5), 1594-1606. https://doi.org/10.1109/TCOMM.2015.2396517

Published in:

IEEE Transactions on Communications

Document Version:

Peer reviewed version

Queen's University Belfast - Research Portal:

Link to publication record in Queen's University Belfast Research Portal

\begin{abstract}
Publisher rights
@ 2015 IEEE. Personal use of this material is permitted. Permission from IEEE must be obtained for all other uses, in any current or future media, including

reprinting/republishing this material for advertising or promotional purposes, creating new collective works, for resale or redistribution to servers or lists, or reuse of any copyrighted component of this work in other works.
\end{abstract}

\section{General rights}

Copyright for the publications made accessible via the Queen's University Belfast Research Portal is retained by the author(s) and / or other copyright owners and it is a condition of accessing these publications that users recognise and abide by the legal requirements associated with these rights.

Take down policy

The Research Portal is Queen's institutional repository that provides access to Queen's research output. Every effort has been made to ensure that content in the Research Portal does not infringe any person's rights, or applicable UK laws. If you discover content in the Research Portal that you believe breaches copyright or violates any law, please contact openaccess@qub.ac.uk. 


\title{
Proactive Relay Selection with Joint Impact of Hardware Impairment and Co-channel Interference
}

\author{
Tran Trung Duy, Trung Q. Duong, Senior Member, IEEE, Daniel Benevides da Costa, Senior Member, IEEE, \\ Vo Nguyen Quoc Bao, Member, IEEE, and Maged Elkashlan, Member, IEEE
}

\begin{abstract}
In this paper, we investigate the end-to-end performance of dual-hop proactive decode-and-forward relaying networks with $N$ th best relay selection in the presence of two practical deleterious effects: i) hardware impairment and ii) cochannel interference. In particular, we derive new exact and asymptotic closed-form expressions for the outage probability and average channel capacity of $N$ th best partial and opportunistic relay selection schemes over Rayleigh fading channels. Insightful discussions are provided. It is shown that, when the system cannot select the best relay for cooperation, the partial relay selection scheme outperforms the opportunistic method under the impact of the same co-channel interference (CCI). In addition, without CCI but under the effect of hardware impairment, it is shown that both selection strategies have the same asymptotic channel capacity. Monte Carlo simulations are presented to corroborate our analysis.
\end{abstract}

Index Terms-Hardware impairment, decode-and-forward relaying, partial relay selection, opportunistic relay selection, outage probability, channel capacity.

\section{INTRODUCTION}

Along the last decade, the concept of cooperative diversity [1] has been well exploited as an efficient means to enhance the performance of wireless communications. The basic idea is to allow single-antenna terminals to share their antennas in order to mimic a physical multiple-antenna array so that spatial diversity can be explored. However, the use of multiple relays may invoke a spectral efficiency loss and relay selection schemes arise as a promising solution for alleviating this problem. Two proactive relay selection strategies ${ }^{1}$ that have been widely investigated in the literature are opportunistic relay selection (ORS) [2]-[11] and partial relay selection (PRS) [12]-[19]. In ORS the best relay is chosen relying on the channel state information (CSI) of both source-relay and relay-destination links. The pioneering idea of ORS was proposed in [2], while [3] presented an asymptotic analysis of the symbol error rate (SER) of a selection amplify-and-forward

Manuscript received April 20, 2014; revised August 14, 2014 and December 15, 2014. The associate editor coordinating the review of this paper and approving it for publication was Prof. Ali Ghrayeb.

T. T. Duy and V. N. Q. Bao are with Posts and Telecommunications Institute of Technology, Vietnam (e-mail: trantrungduy@ ptithcm.edu.vn, baovnq@ptithcm.edu.vn).

T. Q. Duong is with Queen's University Belfast, UK (email: trung.q.duong@qub.ac.uk).

D. B. da Costa is with Federal University of Ceará, CE, Brazil (email: danielbcosta@ieee.org). The work is supported by the CNPq (Grant No. 302106/2011-1)

M. Elkashlan is with Queen Mary University of London, UK (email: maged.elkashlan@qmul.ac.uk).

${ }^{1}$ In proactive relay selection, the relay is chosen before the source transmission.
(AF) network. In [4], it was shown that optimal transmission of a single relay among a set of multiple AF relays minimize the outage probability (OP) and outperform any other strategies that involve simultaneous transmissions from more than one $\mathrm{AF}$ relay under an aggregate power constraint. In [5], the OP of a cooperative network with multiple potential decode-andforward (DF) relays and multiple simultaneous transmissions was investigated, in which a selection cooperation scheme was proposed. In [6], closed-form expressions for the OP and the bit error rate (BER) of uncoded threshold-based ORS were derived assuming arbitrary signal-to-noise ratio (SNR) levels, arbitrary number of available DF relays, and arbitrary sourcedestination channel conditions. In [7], with independent nonidentically distributed (i.n.i.d.) Rician fading channels, approximate formulas for the SER of ORS were derived. Considering i.n.i.d. Nakagami- $m$ fading and a selection combining (SC) receiver at the destination, the outage performance of ORS was examined in [8], while [9] derived closed-form expressions for the SER. In [10], exact closed-form expressions for the OP and ergodic capacity (EC) of selection cooperative relaying were derived assuming a maximal-ratio combiner (MRC) at the destination. In [11], an incremental DF ORS scheme was proposed in which the selected relay chooses to cooperate only if the source-destination channel is of an unacceptable quality. A closed-form expression for the OP was derived.

A common feature of all the aforementioned papers is that full diversity gain can be attained. On the other hand, in these works there is the need for continuous channel feedback from all the links, which results in a high power consumption and large overhead, a non-desirable feature for ad-hoc and sensor networks. To alleviate this problem, PRS was proposed in [12], where only CSI of the source-relay link is used to select the best relay. Thus, by monitoring the connectivity of only one-hop rather than two-hop, the lifetime of the network can be prolonged. In [13], tight closed-form approximations for the EC of dual-hop AF relaying networks with PRS were derived. Relying on the channel quality of the second-hop for selecting the best relay, the work in [14] examined the outage performance of DF relaying networks subject to Nakagami$m$ and employing a MRC receiver at the destination. In [15], a comprehensive performance analysis of dual-hop relaying networks with fixed-gain semi-blind relays was carried out. In particular, closed-form expressions for the OP, probability density function (PDF), moment generating functions (MGFs), and generalized moments of the end-to-end SNR were derived. In addition, the second-order statistics of the end-to-end envelope was studied and the corresponding level crossing rate 
and average fade duration were obtained in an exact manner. In [16], assuming the presence of the direct link between source and destination, an exact performance analysis of DF dual-hop networks with relay selection and subject to i.n.i.d Nakagami$m$ fading was presented. The diversity and coding gains of PRS schemes subject to Nakagami- $m$ fading were attained in [17], while the impact of feedback delay was analyzed in [18]. Finally, in [19], three novel PRS schemes were proposed.

Common to all these works dealing with ORS and PRS is the assumption of perfect transceiver hardware (i.e., ideal hardware) of the terminals. However, in practice, the transceiver hardware is imperfect due to phase noise, I/Q imbalance and amplifier nonlinearities [20]-[22]. Very few works have investigated the effect of hardware impairments in dual-hop cooperative networks and they are briefly discussed next. In [23], the authors quantified the impact of hardware impairments on dual-hop AF and DF relaying networks subject to Nakagami- $m$ fading. Expressing the OP as a function of the effective end-to-end signal-to-noise-and-distortion ratio (SNDR), exact closed-form and asymptotic formulas for the OP were derived considering hardware impairments at the source, relay, and destination. Upper bounds for the EC were derived as well. In that work, fundamental design guidelines for selecting hardware that satisfies the requirements of a practical relaying system were pointed out. In [24], the authors analyzed the impact of hardware impairments at the relay on the OP and the SER in two-way AF relaying.

Another channel impairment that may be taken into account in practical systems is co-channel interference (CCI). Differently from hardware impairments, the study of CCI in cooperative networks has already been extensively investigated along the last years. In the sequel, three representative works will be discussed. In [25], the outage behavior of dual-hop DF ORS schemes was investigated with CCI at both the relays and the destination. It was shown that the co-channel interferers do not affect the diversity gain. However, such interferers degrade the outage performance by affecting the coding gain of the system. In [26], assuming a multiuser relay network composed by a single source, a single AF relay, and multiple destinations, the outage performance of opportunistic scheduling was examined in which the relay and the multiple destinations undergo CCI. Exact expressions and closed-form lower bounds for the OP were derived. In addition, the impact of CSI feedback delay when CCI is considered only at the relay was studied. Finally, in [27], the impact of CCI in twoway AF relaying systems was analyzed.

In this paper, we investigate the end-to-end performance of dual-hop DF relaying networks in the presence of two practical deleterious effects: i) hardware impairment and ii) CCI. Both ORS and PRS schemes are considered. To the best of the authors' knowledge, this is the first attempt to analyze the joint impact of hardware impairment and CCI in a dual-hop relaying network. Assuming Rayleigh fading, new exact and asymptotic closed-form expressions for the OP and the average channel capacity are derived. Insightful discussions are provided. It is shown that, when the system cannot select the best relay for cooperation, PRS scheme outperforms the opportunistic method under the impact of the same CCI. In addition, without

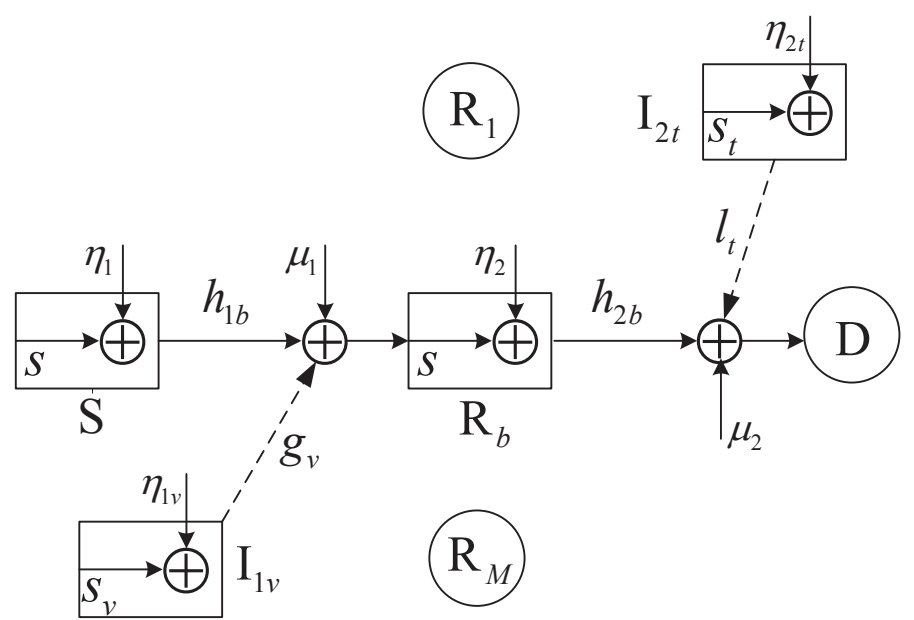

Fig. 1. Dual-hop relay networks in presence of hardware impairments and co-channel interference.

CCI but under the effect of hardware impairment, it is shown that both selection strategies have the same asymptotic channel capacity. Monte Carlo simulations are presented to corroborate our analysis.

The rest of this paper is organized as follows. The system model is described in Section II. In Section III, closed-form expressions for the OP and average channel capacity are derived. Simulation results are presented in Section IV along with representative numerical results. Finally, this paper is concluded in Section V. Appendices A-F present the proofs of the Lemmas and the Theorems.

\section{System/Channel Models AND PREliminary RESULTS}

\section{A. System and Channel Models}

Consider a dual-hop proactive relay network in which a source $S$ attempts to transmit its data to the destination $D$ through the help of $M$ available relays $\mathrm{R}_{m}, m=1,2, \cdots, M$, as shown in Fig. 1. Each terminal is equipped with a single antenna and operates in a half-duplex mode. Assuming that the direct link between $S$ and $D$ experiences deep shadowing, the communication is realized into two time-slots. In our analysis, depending on the available CSI, we consider two well-known proactive relay selection methods: partial relay selection [12] and opportunistic relay selection [28]. In this case, only one relay $\mathrm{R}_{b}$ satisfying a predefined criterion is selected for helping to forward the source message.

In the first time slot, the source transmits its signal $s$ to the chosen relay $\mathrm{R}_{b}$. Assume that there are $K_{1}$ interference sources $\mathrm{I}_{1 v}, v=1,2, \ldots, K_{1}$, which are currently using the same channel, and hence creating interferences to the relay $\mathrm{R}_{b}$. In the second time slot, the relay $\mathrm{R}_{b}$ forwards the source signal to the destination by using a DF protocol. Also, we assume that there are $K_{2}$ interference sources $\mathrm{I}_{2 t}, t=1,2, \ldots, K_{2}$. In the presence of the hardware impairments and co-channel interference, the received signal at $R_{b}$ and $D$ can be expressed, 
respectively, as

$$
\begin{gathered}
y_{\mathrm{R}_{b}}=h_{1 b}\left(s+\eta_{1}\right)+\sum_{v=1}^{K_{1}} g_{v}\left(s_{v}+\eta_{1 v}\right)+\mu_{1}+n_{\mathrm{R}_{b}}, \\
y_{\mathrm{D}}=h_{2 b}\left(s+\eta_{2}\right)+\sum_{t=1}^{K_{2}} l_{t}\left(s_{t}+\eta_{2 t}\right)+\mu_{2}+n_{\mathrm{D}},
\end{gathered}
$$

where $n_{\mathrm{R}_{b}}$ and $n_{\mathrm{D}}$ are, respectively, the additive white Gaussian noise (AWGN) terms at $\mathrm{R}$ and $\mathrm{D}$, with zero mean and variance $N_{0}, s_{v}$ and $s_{t}$ are the signals transmitted by the interference sources $\mathrm{I}_{1 v}$ and $\mathrm{I}_{2 t}$, respectively, $h_{1 b}, h_{2 b}, g_{v}$, and $l_{t}$ are the channel coefficients of the links $\mathrm{S} \rightarrow \mathrm{R}_{b}, \mathrm{R}_{b} \rightarrow \mathrm{D}$, $\mathrm{I}_{1 v} \rightarrow \mathrm{R}_{b}$, and $\mathrm{R}_{b} \rightarrow \mathrm{D}$, respectively. In addition, $\eta_{1}, \eta_{1 v}, \eta_{2}$ and $\eta_{2 t}$ denote the noises caused by the hardware impairments at the transmitters $\mathrm{S}, \mathrm{I}_{1 \mathrm{v}}, \mathrm{R}_{b}$, and $\mathrm{I}_{2 \mathrm{t}}$, respectively, while $\mu_{1}$ and $\mu_{2}$ are the noises generated by the hardware impairments at the receivers $R_{b}$ and $D$, respectively.

Assume that all the channels follow a Rayleigh distribution. Thus, the corresponding channel gains $\varphi_{\mathrm{SR}_{\mathrm{m}}}=\left|h_{1 m}\right|^{2}$, $\varphi_{\mathrm{R}_{\mathrm{m}} \mathrm{D}}=\left|h_{2 m}\right|^{2}$ for $m=1,2, \ldots, M,\left|g_{v}\right|^{2}$, and $\left|l_{t}\right|^{2}$ are exponential random variables (RVs) with parameters $\lambda_{\mathrm{SR}_{m}}$, $\lambda_{\mathrm{R}_{m} \mathrm{D}}, \lambda_{\mathrm{RI}_{1} v}$, and $\lambda_{\mathrm{DI}_{2 t}}$, respectively.

Remark 1: Similar to [23], [24], we can model the distortion noises $\eta_{1}, \eta_{1 v}, \eta_{2}, \eta_{2 t}, \mu_{1}$ and $\mu_{2}$ as circularlysymmetric complex Gaussian distribution with zero-mean and variance $\sigma_{1}^{2} \mathcal{P}_{\mathrm{S}}, \sigma_{3 v}^{2} \mathcal{P}_{\mathrm{l}}, \sigma_{2}^{2} \mathcal{P}_{\mathrm{S}}, \sigma_{4 t}^{2} \mathcal{P}_{\mathrm{l}}, \sigma_{3}^{2}\left(\left|h_{1 b}\right|^{2} \mathcal{P}_{\mathrm{S}}+\left|g_{v}\right|^{2} \mathcal{P}_{\mathrm{l}}\right)$, and $\sigma_{4}^{2}\left(\left|h_{2 b}\right|^{2} \mathcal{P}_{\mathrm{S}}+\left|l_{t}\right|^{2} \mathcal{P}_{\mathrm{I}}\right)$, respectively. In this case, $\mathcal{P}_{\mathrm{S}}$ and $\mathcal{P}_{\mathrm{I}}$ denote the transmit powers of the source (and relays) and the interference sources, respectively, while $\sigma_{1}, \sigma_{3 v}, \sigma_{2}, \sigma_{4 t}$, $\sigma_{3}$ and $\sigma_{4}$ present the level of the hardware impairments at the corresponding transmitters and receivers. Without loss of generality, it is also assumed that all of the nodes have the same structure so that the impairment levels are the same, i.e., $\sigma_{1}=\sigma_{3 v}=\sigma_{4 t}=\sigma_{a}$, and $\sigma_{3}=\sigma_{4}=\sigma_{b}$ [23], [24]. ${ }^{2}$

From (1) and (2), the received signal-to-interference-plusnoise ratio (SINR) at $R_{b}$ and $D$ can be written, respectively, as

$$
\begin{aligned}
\psi_{\mathrm{SR}_{\mathrm{b}}} & =\frac{\mathcal{P}_{\mathrm{S}} \varphi_{\mathrm{SR}_{\mathrm{b}}}}{\left(\sigma_{1}^{2}+\sigma_{3}^{2}\right) \mathcal{P}_{\mathrm{S}} \varphi_{\mathrm{SR}_{\mathrm{b}}}+\sum_{v=1}^{K_{1}}\left(1+\sigma_{1 v}^{2}+\sigma_{3}^{2}\right) \mathcal{P}_{\mathrm{I}}\left|g_{v}\right|^{2}+N_{0}} \\
& =\frac{\gamma_{\mathrm{SR}}}{\kappa \gamma_{\mathrm{SR}_{\mathrm{b}}}+Z_{1}+1},
\end{aligned}
$$

$$
\begin{aligned}
\psi_{\mathrm{R}_{\mathrm{b}} \mathrm{D}} & =\frac{\mathcal{P}_{\mathrm{S}} \varphi_{\mathrm{R}_{\mathrm{b}} \mathrm{D}}}{\left(\sigma_{2}^{2}+\sigma_{4}^{2}\right) \mathcal{P}_{\mathrm{S}} \varphi_{\mathrm{R}_{\mathrm{b}} \mathrm{D}}+\sum_{t=1}^{K_{2}}\left(1+\sigma_{2 t}^{2}+\sigma_{4}^{2}\right) \mathcal{P}_{\mathrm{I}}\left|l_{t}\right|^{2}+N_{0}} \\
& =\frac{\gamma_{\mathrm{R}_{\mathrm{b}} \mathrm{D}}}{\kappa \gamma_{\mathrm{R}_{\mathrm{b}} \mathrm{D}}+Z_{2}+1},
\end{aligned}
$$

\footnotetext{
${ }^{2}$ In case of different levels of hardware impairment, our results can be applied to derive the upper-bound and/or lower-bound of the outage probability and average channel capacity. Moreover, in practice, with knowledge of impairment transceiver levels, we should select the transceivers with similar impairment levels, in order to optimize the system performance (see [23, Corollary 3].
}

where

$$
\begin{aligned}
& \gamma_{\mathrm{SR}}=\frac{\mathcal{P}_{\mathrm{S}} \varphi_{\mathrm{SR}}}{N_{0}}, \gamma_{\mathrm{R}_{\mathrm{b}} \mathrm{D}}=\frac{\mathcal{P}_{\mathrm{S}} \varphi_{\mathrm{R}_{\mathrm{b}} \mathrm{D}}}{N_{0}}, Z_{1}=\sum_{v=1}^{K_{1}}(1+\kappa) \frac{\mathcal{P}_{\mathbf{l}}\left|g_{v}\right|^{2}}{N_{0}}, \\
& Z_{2}=\sum_{t=1}^{K_{2}}(1+\kappa) \frac{\mathcal{P}_{\mathrm{l}}\left|l_{t}\right|^{2}}{N_{0}}, \kappa=\sigma_{a}^{2}+\sigma_{b}^{2} .
\end{aligned}
$$

\section{B. Preliminary Results}

In partial relay selection method, the $N$ th best relay $\mathrm{R}_{b}$ is selected by the following strategy:

$$
\mathrm{R}_{b}=N \text { th } \underset{m=1,2, \ldots, M}{\operatorname{argmax}}\left(\varphi_{\mathrm{SR}_{\mathrm{m}}}\right) .
$$

On the other hand, in the opportunistic relay selection strategy, the $N$ th best relay $\mathrm{R}_{b}$ is chosen according to

$$
\mathrm{R}_{b}=N \text { th } \underset{m=1,2, \ldots M}{\operatorname{argmax}} \min \left(\varphi_{\mathrm{SR}_{\mathrm{m}}}, \varphi_{\mathrm{R}_{\mathrm{m}} \mathrm{D}}\right)
$$

We can observe from (5) and (6) that the relay selection process in the ORS protocol requires each relay to obtain the channel state information (CSI) of the $\mathrm{S} \rightarrow \mathrm{R}$ and $\mathrm{R} \rightarrow \mathrm{D}$ links, while that in the PRS only needs the CSI of the first link. Hence, the implementation of the ORS protocol is more complex than that of the PRS protocol. Moreover, we note that the relay selection operation in the ORS protocol can be realized by a distributed manner as presented in [2].

Remark 2: Throughout this paper, we assume clustering relay networks where data links are independent and identically distributed (i.i.d.), i.e., $\lambda_{\mathrm{SR}_{m}}=\lambda_{\mathrm{SR}}$ and $\lambda_{\mathrm{R}_{m} \mathrm{D}}=\lambda_{\mathrm{RD}}$ for all $m$. In addition, since the interferers can originate from different cells, the interference links are presumed to be independent non-identically distributed (i.n.i.d.), i.e., $\lambda_{\mathrm{RI}_{1 m}} \neq \lambda_{\mathrm{RI}_{1 n}}$ if $m \neq n$, and $\lambda_{\mathrm{DI}_{2 m}} \neq \lambda_{\mathrm{DI}_{2 n}}$ if $m \neq n .^{3}$

The PDF of $Z_{\mathrm{a}}, \mathrm{a} \in\{1,2\}$, can be expressed as

$$
f_{Z_{a}}\left(z_{a}\right)=\sum_{u=1}^{K_{a}} \alpha_{\mathrm{XI}_{a u}} \exp \left(-\Omega_{\mathrm{XI}_{a u}}\right) \text {, }
$$

where $\mathrm{X} \equiv \mathrm{R}$ if $a=1, \mathrm{X} \equiv \mathrm{D}$ if $a=2$,

$$
\begin{aligned}
\Omega_{\mathrm{XI}_{\mathrm{au}}} & =\frac{\tilde{\Omega}_{\mathrm{XI}_{\mathrm{au}}}}{\bar{\gamma}}, \tilde{\Omega}_{\mathrm{XI}_{\mathrm{au}}}=\frac{\lambda_{\mathrm{XI}_{\mathrm{au}}}}{(1+\kappa) r_{P}}, \\
\bar{\gamma} & =\frac{\mathcal{P}_{\mathrm{I}}}{N_{0}}=\frac{\mathcal{P}_{\mathrm{S}}}{N_{0}}=\frac{\mathcal{P}}{N_{0}}, \quad \alpha_{\mathrm{XI}_{\mathrm{au}}}=\frac{\tilde{\alpha}_{\mathrm{XI}_{\mathrm{au}}}}{\bar{\gamma} r_{P}}, \\
\tilde{\alpha}_{\mathrm{XI}_{\mathrm{au}}} & =\tilde{\Omega}_{\mathrm{XI}_{\mathrm{au}}} \prod_{w=1, w \neq u}^{K_{\mathrm{a}}} \frac{\tilde{\Omega}_{\mathrm{XI}_{\mathrm{aw}}}}{\tilde{\Omega}_{\mathrm{XI}_{\mathrm{aw}}}-\tilde{\Omega}_{\mathrm{XI}_{\mathrm{au}}}} .
\end{aligned}
$$

Since the DF relaying protocol is employed, the end-to-end SINR is given by

$$
\psi_{e 2 e}^{\mathrm{Y}}=\min \left(\psi_{\mathrm{SR}_{b}}, \psi_{\mathrm{R}_{b} \mathrm{D}}\right),
$$

where $\mathrm{Y} \in(\mathrm{ORS}, \mathrm{PRS})$.

\footnotetext{
${ }^{3}$ Our derivation can be easily extended to i.n.i.d. data links and/or i.i.d. interference links.
} 


\section{Performance Analysis}

\section{A. Outage Probability}

In this subsection, exact closed-form expressions for the OP of both PRS and ORS schemes will be derived. By definition, the OP is the probability that the end-to-end received SINR is lower than a pre-determined threshold $\gamma_{t h}$.

1) Partial Relay Selection (PRS): The outage probability of the PRS protocol can be formulated as

$$
P_{\mathrm{PRS}}^{\text {out }}=\operatorname{Pr}\left(\psi_{e 2 e}^{\mathrm{PRS}}<\gamma_{t h}\right)=F_{\psi_{e 2 e}^{\mathrm{PRS}}}\left(\gamma_{t h}\right),
$$

where $F_{\psi_{e 2 e}^{\mathrm{PRS}}}($.$) denotes the CDF of \psi_{e 2 e}^{\mathrm{PRS}}$.

Theorem 1: If $x \geq \kappa^{-1}$, then $F_{\psi_{2 e}^{\mathrm{PRS}}}(x)=1$, and if $x<$ $\kappa^{-1}$, it follows that

$$
\begin{aligned}
F_{\psi_{e 2 e}^{\mathrm{PRS}}}(x) & =1-\sum_{m=1}^{N} \sum_{n=0, m+n>1}^{M-m+1} \sum_{v=1}^{K_{1}} \sum_{t=1}^{K_{2}}(-1)^{n+1} \Delta_{1} \Delta_{2} \\
& \times \frac{(1-\kappa x)^{2}}{\left(\Phi_{1}+x\right)\left(\Phi_{2}+x\right)} \exp \left(-\frac{\left(\Theta_{1}+\Omega_{2}\right) x}{1-\kappa x}\right),
\end{aligned}
$$

where $\Omega_{1}=N_{0} \lambda_{\mathrm{SR}} / \mathcal{P}, \quad C_{b}^{a}=\frac{b !}{a !(b-a) !}, \Theta_{1}=$ $(n+m-1) \Omega_{1}, \Delta_{1}=C_{M}^{m-1} C_{M-m+1}^{n} \alpha_{\mathrm{RI}_{1 \mathrm{v}}} /\left(\Theta_{1}-\kappa \Omega_{\mathrm{RI}_{1 \mathrm{v}}}\right)$, $\Phi_{1}=\Omega_{\mathrm{RI}_{1 \mathrm{v}}} /\left(\Theta_{1}-\kappa \Omega_{\mathrm{RI}_{1 \mathrm{v}}}\right), \Omega_{2}=\lambda_{\mathrm{RD}} N_{0} / \mathcal{P}, \Delta_{2}=$ $\alpha_{\mathrm{DI}_{2 \mathrm{t}}} /\left(\Omega_{2}-\Omega_{\mathrm{DI}_{2 \mathrm{t}}} \kappa\right)$ and $\Phi_{2}=\Omega_{\mathrm{DI}_{2 \mathrm{t}}} /\left(\Omega_{2}-\kappa \Omega_{\mathrm{DI}_{2 \mathrm{t}}}\right)$.

Proof 1: The proof is presented in Appendix A.

Lemma 1: Without interference sources, i.e., by setting $\mathcal{P}_{I} \rightarrow 0$ or $r_{P} \rightarrow 0$, and $x<\kappa^{-1}$, the $\mathrm{CDF} F_{\psi_{e 2 e}^{\mathrm{PRS}}}(\cdot)$ can be expressed as

$$
\begin{aligned}
F_{\psi_{e 2 e}^{\mathrm{PRS}}}(x) & =1-\sum_{m=1}^{N} \sum_{n=0, m+n \neq 1}^{M-m+1}(-1)^{n+1} C_{M}^{m-1} C_{M-m+1}^{n} \\
& \times \exp \left(-\frac{\left(\Theta_{1}+\Omega_{2}\right) x}{1-\kappa x}\right) .
\end{aligned}
$$

Proof 2: The proof is given in Appendix B.

Theorem 2: At high transmit SNR and assuming $x<\kappa^{-1}$, the $\mathrm{CDF} F_{\psi_{e 2 e}^{\mathrm{PRS}}}(\cdot)$ can be approximated by

$$
\begin{gathered}
F_{\psi_{e 2 e}^{\mathrm{PRS}}}(x) \stackrel{\bar{\gamma} \rightarrow+\infty}{\approx} 1-\sum_{m=1}^{N} \sum_{n=0, m+n>1}^{M-m+1} \sum_{v=1}^{K_{1}} \sum_{t=1}^{K_{2}}(-1)^{n+1} \\
\times \Delta_{1} \Delta_{2} \frac{(1-\kappa x)^{2}}{\left(\Phi_{1}+x\right)\left(\Phi_{2}+x\right)} .
\end{gathered}
$$

Proof 3: For high values of $\bar{\gamma},(3)$ and (4) can be approximated by

$$
\begin{gathered}
\psi_{\mathrm{SR}_{\mathrm{b}}} \stackrel{\bar{\gamma} \rightarrow+\infty}{\approx} \frac{\gamma_{\mathrm{SR}_{\mathrm{b}}}}{\kappa \gamma_{\mathrm{SR}_{\mathrm{b}}}+Z_{1}}, \\
\psi_{\mathrm{R}_{\mathrm{b}} \mathrm{D}} \stackrel{\bar{\gamma} \rightarrow+\infty}{\approx} \frac{\gamma_{\mathrm{R}_{\mathrm{b}} \mathrm{D}}}{\kappa \gamma_{\mathrm{R}_{\mathrm{b}} \mathrm{D}}+Z_{2}} .
\end{gathered}
$$

From (13), with the same manner with Appendix A, we can obtain

$$
\begin{aligned}
& F_{\psi_{\mathrm{SR}_{\mathrm{b}}}}(x) \stackrel{\bar{\gamma} \rightarrow+\infty}{\approx} 1-\sum_{m=1}^{N} \sum_{n=0, m+n>1}^{M-m+1} \sum_{v=1}^{K_{1}}(-1)^{n+1} \Delta_{1} \frac{1-\kappa x}{\Phi_{1}+x}, \\
& F_{\psi_{\mathrm{R}_{\mathrm{b}} \mathrm{D}}}(x) \stackrel{\bar{\gamma} \rightarrow+\infty}{\approx} 1-\sum_{t=1}^{K_{2}} \Delta_{2} \frac{1-\kappa x}{\Phi_{2}+x} .
\end{aligned}
$$

By substituting the results above into (A.1), (12) can be attained.

Then, similar to Appendix A, (12) can be attained.

Lemma 2: Without interference sources, i.e., by setting $\mathcal{P}_{I} \rightarrow 0$ or $r_{P} \rightarrow 0$, and $x<\kappa^{-1}$, the $\mathrm{CDF} F_{\psi_{e 2 e}^{\mathrm{PRS}}}(\cdot)$ at high transmit SNR can be expressed as

$$
\begin{aligned}
& F_{\psi_{e 2 e}^{\mathrm{PRS}}(x) \stackrel{\bar{\gamma} \rightarrow+\infty}{\approx}}\left\{\begin{array}{ll}
\Omega_{2} x /(1-\kappa x) ; & \text { if } N<M \\
\left(M \Omega_{1}+\Omega_{2}\right) x /(1-\kappa x) & \text { if } N=M
\end{array} .\right.
\end{aligned}
$$

Proof 4: The proof is given in Appendix C.

From Lemma 2, one can observe that when $x<\kappa^{-1}$, the diversity order equals 1 .

2) Opportunistic Relay Selection (ORS): The OP of the ORS scheme can be formulated as

$$
P_{\mathrm{ORS}}^{\text {out }}=\operatorname{Pr}\left(\psi_{e 2 e}^{\mathrm{ORS}}<\gamma_{t h}\right)=F_{\psi_{e 2 e}^{\mathrm{ORS}}}\left(\gamma_{t h}\right),
$$

Theorem 3: If $x \geq \kappa^{-1}$, then $F_{\psi_{e 2 e}^{\mathrm{ORS}}}(x)=1$, and if $x<$ $\kappa^{-1}$, it follows that

$$
\begin{aligned}
F_{\psi_{e 2 e}^{\mathrm{ORS}}}(x) & =1-\sum_{m=1}^{N} \sum_{n=0, m+n>1}^{M-m+1} \sum_{v=1}^{K_{1}} \sum_{t=1}^{K_{2}}(-1)^{n+1} \\
& \times\left[\frac{\Delta_{3}}{\Phi_{3}+x}+\frac{\Delta_{4}}{\Phi_{4}+x}+\frac{\Delta_{5}}{\Phi_{6}+x}+\frac{\Delta_{6}}{\Phi_{7}+x}\right] \\
& \times \frac{(1-\kappa x)^{2}}{\left(\Phi_{5}+x\right)} \exp \left(-\frac{\Theta_{2} x}{1-\kappa x}\right)
\end{aligned}
$$

where $\Omega=\Omega_{1}+\Omega_{2}, \Theta_{2}=(n+m-1) \Omega$, $\Phi_{3}=\Omega_{\mathrm{RI}_{1 \mathrm{v}}} /\left(\Omega_{1}-\kappa \Omega_{\mathrm{RI}_{1 \mathrm{v}}}\right), \Phi_{4}=\Omega_{\mathrm{RI}_{1 \mathrm{v}}} /\left(\Theta_{2}-\kappa \Omega_{\mathrm{RI}_{1 \mathrm{v}}}\right)$, $\Phi_{5}=\left(\Omega_{\mathrm{RI}_{1 \mathrm{v}}}+\Omega_{\mathrm{DI}_{2 \mathrm{t}}}\right) /\left(\Theta_{2}-\kappa\left(\Omega_{\mathrm{RI}_{1 \mathrm{v}}}+\Omega_{\mathrm{DI}_{2 \mathrm{t}}}\right)\right)$, $\Phi_{6}=\Omega_{\mathrm{DI}_{2 \mathrm{t}}} /\left(\Omega_{2}-\kappa \Omega_{\mathrm{DI}_{2 \mathrm{t}}}\right), \Phi_{7}=\Omega_{\mathrm{DI}_{2 \mathrm{t}}} /\left(\Theta_{2}-\kappa \Omega_{\mathrm{DI}_{2 \mathrm{t}}}\right)$,

$$
\begin{aligned}
\Delta_{3} & =(n+m-1) C_{M}^{m-1} C_{M-m+1}^{n} \frac{\Omega_{2} \alpha_{\mathrm{RI}_{1 \mathrm{v}}} \alpha_{\mathrm{DI}_{2 \mathrm{t}}}}{\Omega_{2}+(n+m-2) \Omega} \\
& \times \frac{1}{\left(\Theta_{2}-\kappa\left(\Omega_{\mathrm{RI}_{1 \mathrm{v}}}+\Omega_{\mathrm{DI}_{2 \mathrm{t}}}\right)\right)\left(\Omega_{1}-\kappa \Omega_{\mathrm{RI}_{1 \mathrm{v}}}\right)}, \\
\Delta_{4} & =(n+m-2) C_{M}^{m-1} C_{M-m+1}^{n} \frac{\Omega_{1} \alpha_{\mathrm{RI}_{1 \mathrm{v}}} \alpha_{\mathrm{DI}_{2 \mathrm{t}}}}{\Omega_{2}+(n+m-2) \Omega} \\
& \times \frac{1}{\left(\Theta_{2}-\kappa \Omega_{\mathrm{RI}_{1 \mathrm{v}}}\right)\left(\Theta_{2}-\kappa\left(\Omega_{\mathrm{RI}_{1 \mathrm{v}}}+\Omega_{\mathrm{DI}_{2 \mathrm{t}}}\right)\right)}, \\
\Delta_{5} & =(n+m-1) C_{M}^{m-1} C_{M-m+1}^{n} \frac{\Omega_{1} \alpha_{\mathrm{RI}_{1 \mathrm{v}}} \alpha_{\mathrm{DI}_{2 \mathrm{t}}}}{\Omega_{1}+(n+m-2) \Omega} \\
& \times \frac{1}{\left(\Theta_{2}-\kappa\left(\Omega_{\mathrm{RI}_{1 \mathrm{v}}}+\Omega_{\mathrm{DI}_{2 \mathrm{t}}}\right)\right)\left(\Omega_{2}-\kappa \Omega_{\mathrm{DI}_{2 \mathrm{t}}}\right)}, \\
\Delta_{6} & =(n+m-2) C_{M}^{m-1} C_{M-m+1}^{n} \frac{\Omega_{2} \alpha_{\mathrm{RI}_{1 \mathrm{v}}} \alpha_{\mathrm{DI}_{2 \mathrm{t}}}}{\Omega_{1}+(n+m-2) \Omega} \\
& \times \frac{1}{\left(\Theta_{2}-\kappa \Omega_{\mathrm{DI}_{2 \mathrm{t}}}\right)\left(\Theta_{2}-\kappa\left(\Omega_{\mathrm{RI}_{1 \mathrm{v}}}+\Omega_{\mathrm{DI}_{2 \mathrm{t}}}\right)\right)} .
\end{aligned}
$$

Proof 5: The proof is presented in Appendix D.

Lemma 3: Without interference sources, i.e., by setting $\mathcal{P}_{I} \rightarrow 0$ or $r_{P} \rightarrow 0$, and $x<\kappa^{-1}$, the $\mathrm{CDF} F_{\psi_{e 2 e}^{\mathrm{ORS}}}^{\mathrm{OR}}(\cdot)$ can be expressed as

$$
\begin{aligned}
F_{\psi_{e 2 e}^{\mathrm{ORS}}}^{\mathrm{ORS}}(x) & =1-\sum_{m=1}^{N} \sum_{n=0, n+m>1}^{M-m+1}(-1)^{n+1} C_{M}^{m-1} C_{M-m+1}^{n} \\
& \times \exp \left(-(n+m-1) \frac{\Omega x}{1-\kappa x}\right) .
\end{aligned}
$$


Proof 6: From (D.3), (17) can be obtained.

Theorem 4: At high transmit SNR and assuming $x<\kappa^{-1}$, the $\mathrm{CDF} F_{\psi_{e 2 e}^{\mathrm{PRS}}}(\cdot)$ can be approximated by

$$
\begin{aligned}
F_{\psi_{e 2 e}^{\mathrm{ORS}}}(x) & \stackrel{\bar{\gamma} \rightarrow+\infty}{\approx} 1-\sum_{m=1}^{N} \sum_{n=0, m+n>1}^{M-m+1} \sum_{v=1}^{K_{1}} \sum_{t=1}^{K_{2}}(-1)^{n+1} \\
& \times\left[\frac{\Delta_{3}}{\Phi_{3}+x}+\frac{\Delta_{4}}{\Phi_{4}+x}+\frac{\Delta_{5}}{\Phi_{6}+x}+\frac{\Delta_{6}}{\Phi_{7}+x}\right] \\
& \times \frac{(1-\kappa x)^{2}}{\Phi_{5}+x} .
\end{aligned}
$$

Proof 7: Note that, for high $\bar{\gamma}$ values, (3) and (4) can be approximated by (13). Hence, similar as obtained (13) from (12), (18) can be attained by omitting the term $\exp \left(-\frac{\Theta_{2} x}{1-\kappa x}\right)$ from (16).

Lemma 4: Without interference sources and considering $x<$ $\kappa^{-1}$, the $\mathrm{CDF} F_{\psi_{e 2 e}^{\mathrm{ORS}}}(\cdot)$ at high transmit SNR can be written as

$$
F_{\gamma_{1 b}}(x) \stackrel{\bar{\gamma} \rightarrow+\infty}{\approx} C_{M}^{N-1}\left(\frac{\Omega x}{1-\kappa x}\right)^{M-N+1} .
$$

Proof 8: The proof is similar to that of Lemma 2.

From (19), one can attest that the diversity order of the ORS strategy equals to $M-N+1$.

\section{B. Average Channel Capacity}

The average channel capacity can be mathematically defined as

$$
\begin{aligned}
C_{\text {avg }}^{\mathrm{Y}} & =\frac{1}{2} \mathcal{E}\left\{\log _{2}\left(1+\psi_{e 2 e}^{\mathrm{Y}}\right)\right\} \\
& =\frac{1}{2 \ln 2} \int_{0}^{\kappa^{-1}} \ln (1+x) f_{\psi_{e 2 e}^{\mathrm{Y}}}(x) d x,
\end{aligned}
$$

where $\mathrm{Y} \in\{\mathrm{PRS}, \mathrm{ORS}\}, \mathcal{E}\{$.$\} symbolizes expectation, and$ $f_{\psi_{e 2 e}^{\mathrm{Y}}}(\cdot)$ denotes the PDF of $\psi_{e 2 e}^{\mathrm{Y}}$.

From (10) and (16), (20) can be rewritten as

$$
C_{a v g}^{\mathrm{Y}}=\frac{1}{2 \ln 2} \int_{0}^{\kappa^{-1}} \frac{1-F_{\psi_{e 2 e}^{\mathrm{Y}}}(x)}{1+x} d x .
$$

Proposition 1: In the presence of hardware impairments, i.e., $\kappa>0$, the average channel capacity of both PRS and ORS methods is bounded by

$$
C_{\text {avg }}^{\mathrm{A}} \leq \frac{1}{2 \ln 2} \ln \left(1+\frac{1}{\kappa}\right) .
$$

Proof 9: From (3) and (4), it is easy to see that $\psi_{1 b} \leq$ $\kappa^{-1}$ and $\psi_{2 b} \leq \kappa^{-1}$, which implies in $\psi_{e 2 e}^{\mathrm{Y}} \leq \kappa^{-1}$. Thus, combining with (20), (22) can be readily obtained.

Before calculating the average capacity of the PRS and ORS strategies, the following integral will be introduced.

$$
\begin{aligned}
\mathcal{J}(\kappa, \Omega, \Phi) & =\int_{0}^{\kappa^{-1}} \frac{1}{\Phi+x} \exp \left(-\frac{\Omega x}{1-\kappa x}\right) d x \\
& =\exp \left(\frac{\Omega \Phi}{\Phi \kappa+1}\right) E_{1}\left(\frac{\Omega \Phi}{\Phi \kappa+1}\right) \\
& -\exp \left(\frac{\Omega}{\kappa}\right) E_{1}\left(\frac{\Omega}{\kappa}\right),
\end{aligned}
$$

where $E_{1}($.$) denotes the exponential integral function [29].$

Proof 10: By interchanging the variable $t=1 /(1-\kappa x)$, $\mathcal{J}(\kappa, \Omega, \Phi)$ can be rewritten as

$$
\begin{aligned}
\mathcal{J}(\kappa, \Omega, \Phi) & =\frac{\exp (\Omega / \kappa)}{\kappa \Phi+1} \int_{1}^{+\infty} \frac{\exp (-t \Omega / \kappa)}{t(t-1 /(\kappa \Phi+1))} d t \\
& =\exp \left(\frac{\Omega \Phi}{\kappa \Phi+1}\right) \int_{\frac{\kappa \Phi}{\kappa \Phi+1}}^{+\infty} \frac{\exp (-t \Omega / \kappa)}{t} d t \\
& -\exp \left(\frac{\Omega}{\kappa}\right) \int_{1}^{+\infty} \frac{\exp (-t \Omega / \kappa)}{t} d t .
\end{aligned}
$$

Then, by using the definition of the exponential integral function $E_{1}(x)=\int_{x}^{+\infty} \frac{\exp (-t)}{t} d t$, we can easily obtain (23).

1) Partial Relay Selection (PRS):

Theorem 5: The average channel capacity of the PRS method can be expressed as (24), shown at the top of next page, with $\delta_{1}=\left(1+\kappa \Phi_{1}\right)^{2} /\left(\Phi_{2}-\Phi_{1}\right)$ and $\delta_{2}=$ $\left(1+\kappa \Phi_{2}\right)^{2} /\left(\Phi_{1}-\Phi_{2}\right)$.

Proof 11: The proof is presented in Appendix E.

Lemma 5: Without interference sources, the average channel capacity of the PRS method is given by

$$
\begin{aligned}
C_{\text {avg }}^{\mathrm{PRS}} & =\frac{1}{2 \ln 2} \sum_{m=1}^{N} \sum_{n=0, m+n>1}^{M-m+1} \sum_{v=1}^{K_{1}} \sum_{t=1}^{K_{2}}(-1)^{n+1} \\
& \times \Delta_{1} \Delta_{2} \mathcal{J}\left(\kappa, \Theta_{1}+\Omega_{2}, 1\right) .
\end{aligned}
$$

Proof 12: Relying on (1), (21), and (23), Lemma 5 can be easily proved.

Theorem 6: At high transmit SNR $\bar{\gamma}$, the asymptotic average channel capacity of the PRS method can be derived as (26), shown at the top of next page.

Proof 13: (26) can be attained from (24) by performing the appropriate substitutions, i.e., replacing $\mathcal{J}\left(\kappa, \Theta_{1}+\Omega_{2}, 1\right)$, $\mathcal{J}\left(\kappa, \Theta_{1}+\Omega_{2}, \Phi_{1}\right)$, and $\mathcal{J}\left(\kappa, \Theta_{1}+\Omega_{2}, \Phi_{2}\right)$ by $\mathcal{J}(\kappa, 0,1)$, $\mathcal{J}\left(\kappa, 0, \Phi_{1}\right)$ and $\mathcal{J}\left(\kappa, 0, \Phi_{1}\right)$, respectively. In addition, note that $\mathcal{J}(\kappa, 0, \Omega)=\ln ((1+\kappa \Phi) / \kappa \Phi)$.

Finally, one can see that without interference sources, the asymptotic average capacity of the PRS method is given as in (22), i.e.,

$$
C_{\text {avg }}^{\mathrm{PRS}} \stackrel{\bar{\gamma} \rightarrow+\infty}{\approx} \frac{1}{2 \ln 2} \ln \left(1+\frac{1}{\kappa}\right) .
$$

\section{2) Opportunistic Relay Selection (ORS):}

Theorem 7: The average channel capacity of the ORS method can be given as (28), shown at the top of next page, with $\delta_{3}=\left(1+\kappa \Phi_{3}\right)^{2} /\left(\Phi_{5}-\Phi_{3}\right), \quad \delta_{4}=$ $\left(1+\kappa \Phi_{5}\right)^{2} /\left(\Phi_{3}-\Phi_{5}\right), \delta_{5}=\left(1+\kappa \Phi_{4}\right)^{2} /\left(\Phi_{5}-\Phi_{4}\right), \delta_{6}=$ $\left(1+\kappa \Phi_{5}\right)^{2} /\left(\Phi_{4}-\Phi_{5}\right), \delta_{7}=\left(1+\kappa \Phi_{6}\right)^{2} /\left(\Phi_{5}-\Phi_{6}\right), \delta_{8}=$ $\left(1+\kappa \Phi_{5}\right)^{2} /\left(\Phi_{6}-\Phi_{5}\right), \delta_{9}=\left(1+\kappa \Phi_{7}\right)^{2} /\left(\Phi_{5}-\Phi_{7}\right)$, and $\delta_{10}=\left(1+\kappa \Phi_{5}\right)^{2} /\left(\Phi_{7}-\Phi_{5}\right)$.

Proof 14: The proof is presented in Appendix F .

Lemma 6: Without interference sources, the average channel capacity can be rewritten as

$$
\begin{aligned}
C_{a v g}^{\text {ORS }} & =\sum_{m=1}^{N} \sum_{n=0, n+m>1}^{M-m+1}(-1)^{n} C_{M}^{m-1} C_{M-m+1}^{n} \\
& \times \mathcal{J}(\kappa,(n+m-1) \Omega x, 1) .
\end{aligned}
$$




$$
\begin{aligned}
C_{\text {avg }}^{\mathrm{PRS}} & =\frac{1}{2 \ln 2} \sum_{m=1}^{N} \sum_{n=0, m+n>1}^{M-m+1} \sum_{v=1}^{K_{1}} \sum_{t=1}^{K_{2}}(-1)^{n+1} \Delta_{1} \Delta_{2} \\
& \times\left[\left(\frac{\delta_{1}}{\Phi_{1}-1}+\frac{\delta_{2}}{\Phi_{2}-1}+\kappa^{2}\right) \mathcal{J}\left(\kappa, \Theta_{1}+\Omega_{2}, 1\right)-\frac{\delta_{1}}{\Phi_{1}-1} \mathcal{J}\left(\kappa, \Theta_{1}+\Omega_{2}, \Phi_{1}\right)-\frac{\delta_{2}}{\Phi_{2}-1} \mathcal{J}\left(\kappa, \Theta_{1}+\Omega_{2}, \Phi_{2}\right)\right]
\end{aligned}
$$

$$
\begin{aligned}
C_{a v g}^{\mathrm{PRS}} & \stackrel{\bar{\gamma} \rightarrow+\infty}{\approx} \frac{1}{2 \ln 2} \sum_{m=1}^{N} \sum_{n=0, m+n>1}^{M-m+1} \sum_{v=1}^{K_{1}} \sum_{t=1}^{K_{2}}(-1)^{n+1} \Delta_{1} \Delta_{2} \\
& \times\left[\left(\frac{\delta_{1}}{\Phi_{1}-1}+\frac{\delta_{2}}{\Phi_{2}-1}+\kappa^{2}\right) \ln \left(\frac{1+\kappa}{\kappa}\right)-\frac{\delta_{1}}{\Phi_{1}-1} \ln \left(\frac{1+\kappa \Phi_{1}}{\kappa \Phi_{1}}\right)-\frac{\delta_{2}}{\Phi_{2}-1} \ln \left(\frac{1+\kappa \Phi_{2}}{\kappa \Phi_{2}}\right)\right]
\end{aligned}
$$

$$
\begin{aligned}
C_{\text {avg }}^{\mathrm{ORS}} & =\frac{1}{2 \ln 2} \sum_{m=1}^{N} \sum_{n=0, m+n>1}^{M-m+1} \sum_{v=1}^{K_{1}} \sum_{t=1}^{K_{2}}(-1)^{n+1} \\
& \times\left(\frac{\Delta_{3} \delta_{3}}{\Phi_{3}-1}+\frac{\Delta_{4} \delta_{5}}{\Phi_{4}-1}+\frac{\Delta_{5} \delta_{7}}{\Phi_{6}-1}+\frac{\Delta_{6} \delta_{9}}{\Phi_{7}-1}+\frac{\Delta_{3} \delta_{4}+\Delta_{4} \delta_{6}+\Delta_{5} \delta_{8}+\Delta_{6} \delta_{10}}{\Phi_{5}-1}+\left(\Delta_{3}+\Delta_{4}\right) \kappa^{2}\right) \mathcal{J}\left(\kappa, \Theta_{2}, 1\right) \\
& -\frac{\Delta_{3} \delta_{3}}{\Phi_{3}-1} \mathcal{J}\left(\kappa, \Theta_{2}, \Phi_{3}\right)-\frac{\Delta_{4} \delta_{5}}{\Phi_{4}-1} \mathcal{J}\left(\kappa, \Theta_{2}, \Phi_{4}\right)-\frac{\Delta_{5} \delta_{7}}{\Phi_{6}-1} \mathcal{J}\left(\kappa, \Theta_{2}, \Phi_{6}\right)-\frac{\Delta_{6} \delta_{9}}{\Phi_{7}-1} \mathcal{J}\left(\kappa, \Theta_{2}, \Phi_{7}\right) \\
& -\left(\frac{\Delta_{3} \delta_{4}+\Delta_{4} \delta_{6}+\Delta_{5} \delta_{8}+\Delta_{6} \delta_{10}}{\Phi_{5}-1}\right) \mathcal{J}\left(\kappa, \Theta_{2}, \Phi_{5}\right)
\end{aligned}
$$

$$
\begin{aligned}
C_{a v g}^{\mathrm{ORS}} & \stackrel{\bar{\gamma}}{\rightarrow} \underset{ }{\approx} \frac{1}{2 \ln 2} \sum_{m=1}^{N} \sum_{n=0, m+n>1}^{M-m+1} \sum_{v=1}^{K_{1}} \sum_{t=1}^{K_{2}}(-1)^{n+1} \\
& \times\left(\frac{\Delta_{3} \delta_{3}}{\Phi_{3}-1}+\frac{\Delta_{4} \delta_{5}}{\Phi_{4}-1}+\frac{\Delta_{5} \delta_{7}}{\Phi_{6}-1}+\frac{\Delta_{6} \delta_{9}}{\Phi_{7}-1}+\frac{\Delta_{3} \delta_{4}+\Delta_{4} \delta_{6}+\Delta_{5} \delta_{8}+\Delta_{6} \delta_{10}}{\Phi_{5}-1}+\left(\Delta_{3}+\Delta_{4}\right) \kappa^{2}\right) \ln \left(\frac{1+\kappa}{\kappa}\right) \\
& -\frac{\Delta_{3} \delta_{3}}{\Phi_{3}-1} \ln \left(\frac{1+\kappa \Phi_{3}}{\kappa \Phi_{3}}\right)-\frac{\Delta_{4} \delta_{5}}{\Phi_{4}-1} \ln \left(\frac{1+\kappa \Phi_{4}}{\kappa \Phi_{4}}\right)-\frac{\Delta_{5} \delta_{7}}{\Phi_{6}-1} \ln \left(\frac{1+\kappa \Phi_{6}}{\kappa \Phi_{6}}\right)-\frac{\Delta_{6} \delta_{9}}{\Phi_{7}-1} \ln \left(\frac{1+\kappa \Phi_{7}}{\kappa \Phi_{7}}\right) \\
& -\left(\frac{\Delta_{3} \delta_{4}+\Delta_{4} \delta_{6}+\Delta_{5} \delta_{8}+\Delta_{6} \delta_{10}}{\Phi_{5}-1}\right) \ln \left(\frac{1+\kappa \Phi_{5}}{\kappa \Phi_{5}}\right) .
\end{aligned}
$$

Proof 15: Based on (17), (21), and (23), Lemma 6 can be readily proved.

Theorem 8: At high transmit SNR $\bar{\gamma}$, the asymptotic average channel capacity of the PRS method can be derived as (30), shown at the top of next page.

Proof 16: The proof of Theorem 8 is similar to that of Theorem 6.

One can easily prove that the asymptotic average capacity of the PRS method at high $\bar{\gamma}$ is given as in (27), i.e.,

$$
C_{\text {avg }}^{\mathrm{ORS}} \stackrel{\bar{\gamma} \rightarrow+\infty}{\approx} \frac{1}{2 \ln 2} \ln \left(1+\frac{1}{\kappa}\right)
$$

From (27) and (31), note that under the impact of hardware impairment and without co-channel interference, the PRS and ORS schemes have the same average channel capacity at high transmit SNR.

\section{Numerical Results AND Simulations}

In this Section, representative numerical results are presented to illustrate the performance of the two proposed relay selection schemes in the presence of hardware impairment and CCI. Monte Carlo simulation results are also shown to corroborate the proposed analysis. Without any loss of generality, we set $\gamma_{t h}<\kappa^{-1}$.

In Fig. 2, the outage probability is plotted as a function of transmit SNR $\bar{\gamma}$. The following parameters are employed: $M=4, K_{1}=K_{2}=2, r_{P}=1, \gamma_{t h}=1, \kappa=0.075, \lambda_{\mathrm{SR}}=$ $0.3, \lambda_{\mathrm{RD}}=0.5, \lambda_{\mathrm{RI}_{1 \mathrm{v}}} \in\{1,2\}$, and $\lambda_{\mathrm{DI}_{2 \mathrm{t}}} \in\{1.5,2.5\}$. It can be observed that the outage performance of the ORS and PRS schemes is better if the system can select the best relay for the cooperation $(N=1)$. In addition, when $N=1$, the outage probability of the ORS scheme is lower than that of the PRS one. However, such a metric of the PRS is higher than that of the ORS when $N=2$. It is because that when the best relay cannot be selected, the end-to-end SINR of the 


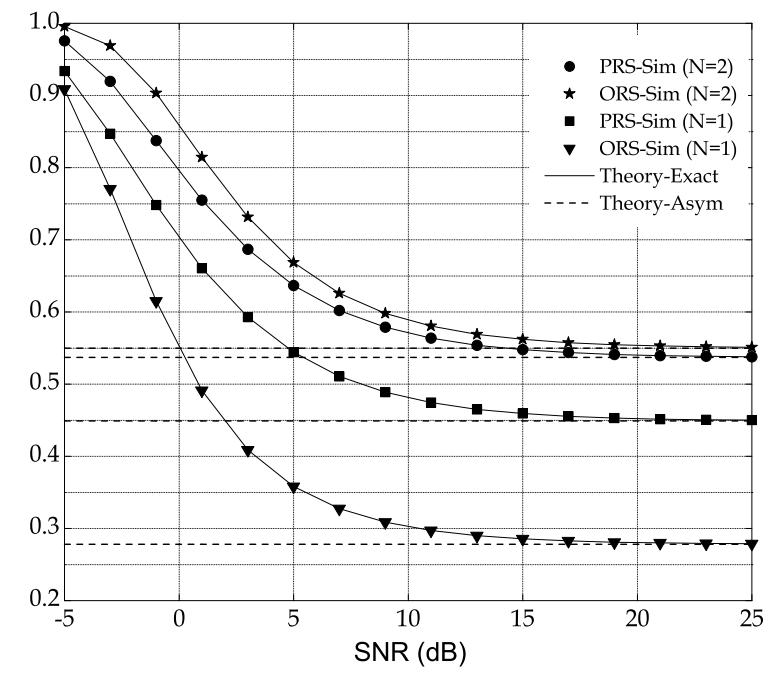

Fig. 2. Outage probability as a function of the transmit SNR $\bar{\gamma}$ when $M=4$, $K_{1}=K_{2}=2, r_{P}=1, \gamma_{t h}=1, \kappa=0.075, \lambda_{\mathrm{SR}}=0.3, \lambda_{\mathrm{RD}}=0.5$, $\lambda_{\mathrm{RI}_{1 \mathrm{v}}} \in\{1,2\}$, and $\lambda_{\mathrm{DI}_{2 \mathrm{t}}} \in\{1.5,2.5\}$.

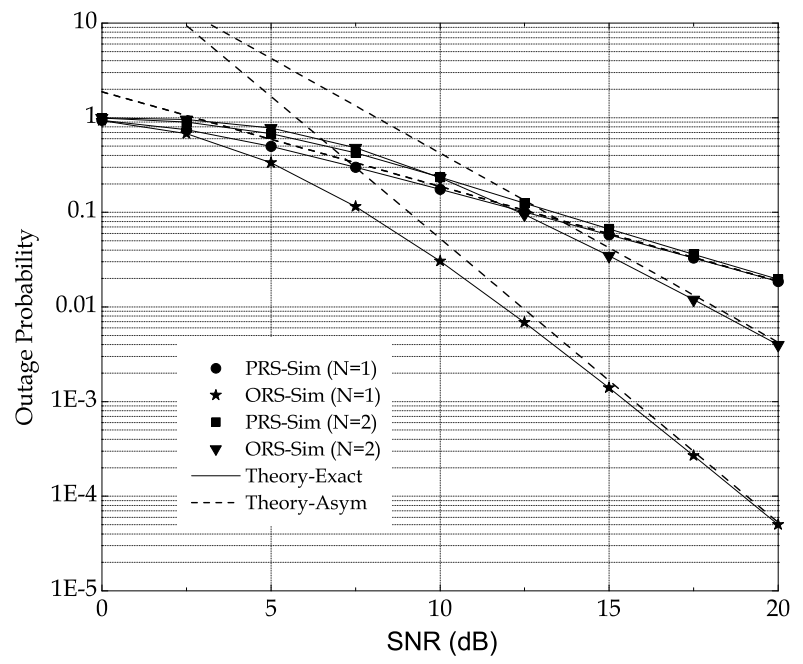

Fig. 3. Outage probability as a function of the transmit SNR $\bar{\gamma}$ when $M=3$, $r_{P}=0, \gamma_{t h}=1.5, \kappa=0.08, \lambda_{\mathrm{SR}}=1.1$, and $\lambda_{\mathrm{RD}}=1.1$.

ORS protocol is no longer maximum. Hence, PRS can provide a higher end-to-end SINR than ORS. Finally, it can be seen that the outage probability decreases when the transmit SNR increases. However, the outage performance of both protocols converges to positive constant at high SNR regime. Therefore, we can conclude that the system obtains the zero-diversity order when there are the interference sources in the network.

In Fig. 3, the outage performance is depicted as a function of transmit SNR $\bar{\gamma}$ when there is no interference source and by setting $M=3, r_{P}=0, \gamma_{t h}=1.5, \kappa=0.08$ and $\lambda_{\mathrm{SR}}=$ $\lambda_{\mathrm{RD}}=1.1$. Note that the ORS scheme outperforms the PRS one for both $N=1,2$, with the performance gap being higher

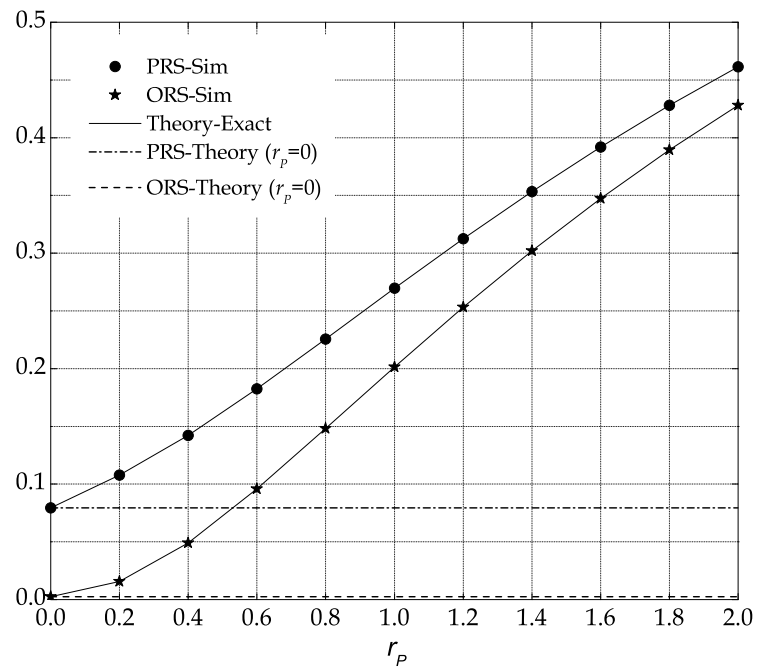

Fig. 4. Outage probability as a function of the ratio $r_{P}$ when $\bar{\gamma}=10 \mathrm{~dB}$, $M=6, N=2, K_{1}=K_{2}=1, \gamma_{t h}=0.5, \kappa=0.08, \lambda_{\mathrm{SR}}=1$, $\lambda_{\mathrm{RD}}=0.5, \lambda_{\mathrm{RI}_{11}}=1.5$, and $\lambda_{\mathrm{DI}_{21}}=2$.

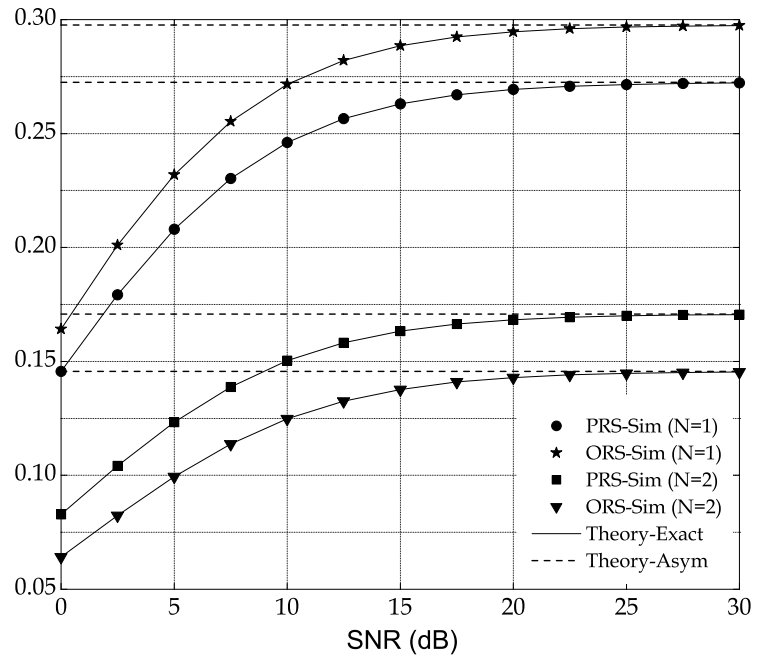

Fig. 5. Average channel capacity as a function of the transmit SNR $\bar{\gamma}$ when $M=2, K_{1}=K_{2}=1, r_{P}=1, \kappa=0.075, \lambda_{\mathrm{SR}}=1.1, \lambda_{\mathrm{RD}}=1.3$, and $\lambda_{\mathrm{RI}_{11}}=\lambda_{\mathrm{DI}_{21}}=0.7$.

for the case $N=1$. The reason is that the diversity $\operatorname{order}^{4}$ of the ORS scheme equals to 3 for $N=1$, while it is 2 for $N=2$. Indeed, for $N=2$, the performance of both schemes is almost the same at low and medium SNRs, and only at high SNR region a practical difference in performance can be detected.

In Fig. 4, we investigate the impact of the ratio $r_{P}\left(\mathcal{P}_{I} / \mathcal{P}_{S}\right)$ on the outage performance of the proposed protocols. For the illustrative purpose, we fix the parameters $\bar{\gamma}, M, N, K_{1}$, $K_{2}, \gamma_{t h}, \kappa, \lambda_{\mathrm{SR}}, \lambda_{\mathrm{RD}}, \lambda_{\mathrm{RI}_{11}}$ and $\lambda_{\mathrm{DI}_{21}}$ by $10 \mathrm{~dB}, 6,2,1$,

\footnotetext{
${ }^{4}$ The diversity order of the PRS scheme is always 1 , regardless of the value of $N$.
} 


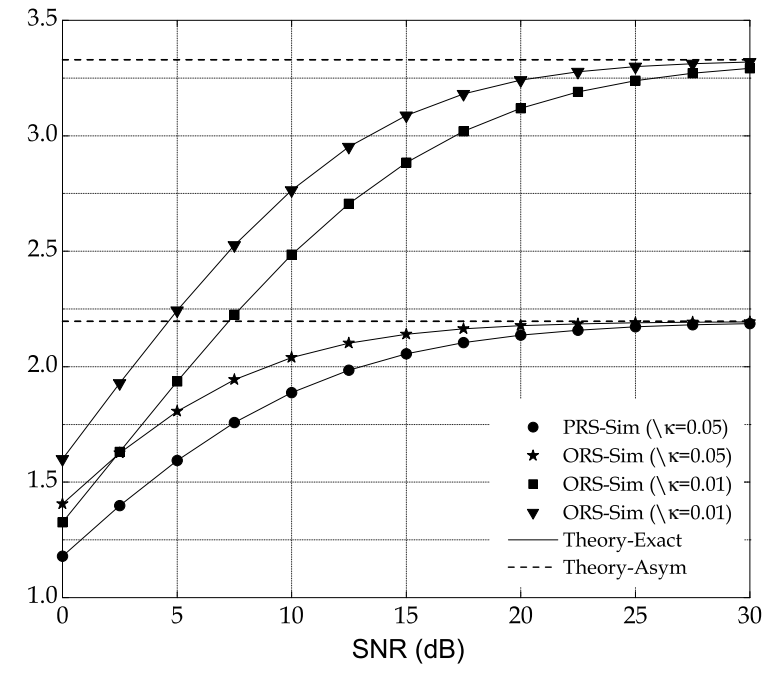

Fig. 6. Average channel capacity as a function of the transmit SNR $\bar{\gamma}$ when $M=4, N=1, r_{P}=0$, and $\lambda_{\mathrm{SR}}=\lambda_{\mathrm{RD}}=0.1$.

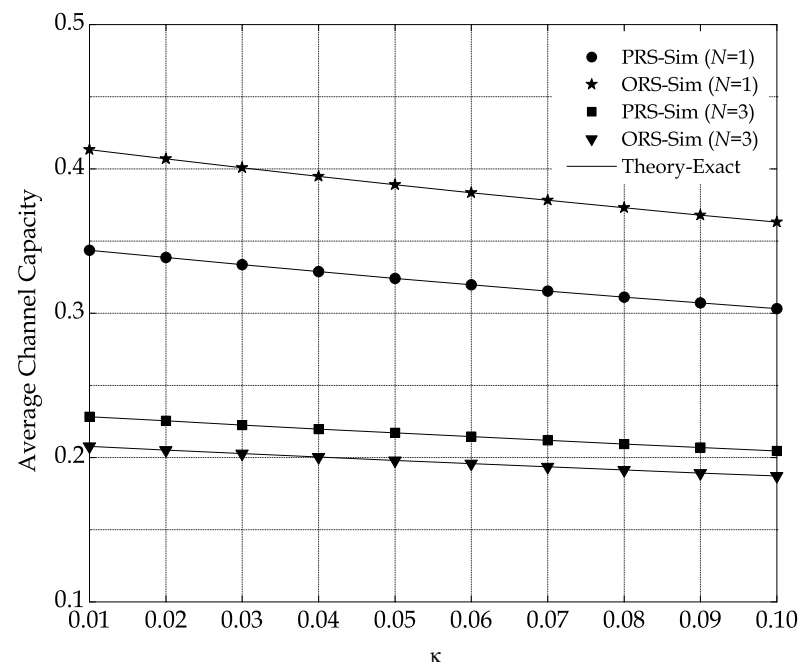

Fig. 7. Average channel capacity as a function of $\kappa$ when $\bar{\gamma}=10 \mathrm{~dB}$ $M=5, K_{1}=K_{2}=1, r_{P}=1, \lambda_{\mathrm{SR}}=\lambda_{\mathrm{RD}}=1, \lambda_{\mathrm{RI}_{11}}=0.5$, and lambda $\mathrm{RI}_{11}=0.75$.

$1,0.5,0.08,1,0.5,1.5$ and 2 , respectively. It can be seen from this figure that the outage performance of both protocols decreases when the ratio $r_{P}$ increases. Different with the results presented in Fig. 2, although the system can only select the second-best relay for the cooperation, the ORS protocol obtains better performance as compared with the PRS protocol.

Fig. 5 presents the average channel capacity of the PRS and ORS protocols as a function of the transmit SNR $\bar{\gamma}$. In this figure, we fix the parameters as follows: $M=2$, $K_{1}=K_{2}=1, r_{P}=1, \kappa=0.075$ and $\lambda_{\mathrm{SR}}=1.1$, $\lambda_{\mathrm{RD}}=1.3$, and $\lambda_{\mathrm{RI}_{11}}=\lambda_{\mathrm{DI}_{21}}=0.7$. Similar to Fig. 2, the ORS scheme achieves higher channel capacity than PRS one when the system can select the best relay (i.e., $N=1$ ) for cooperation. Otherwise, for $N=2$, i.e., the system selects one the second best relay for cooperation, the PRS strategy attains better performance. Finally, note that the channel capacity of both schemes converges to the asymptotic values at high SNR region.

In Fig. 6, the effect of the hardware impairment level $\kappa$ on the average channel capacity is investigated. It is assumed that there is no $\mathrm{CCI}$, i.e., $r_{P}=0$. The remaining parameters are designed as follows: $M=4, N=1$, and $\lambda_{\mathrm{SR}}=\lambda_{\mathrm{RD}}=$ 0.1. One can notice that the PRS and ORS schemes have the same asymptotic channel capacity. In addition, it is shown that both strategies obtain better performance as the value of $\kappa$ decreases, with ORS presenting better performance than PRS.

Fig. 7 presents the average channel capacity as a function of $\kappa$ when $\bar{\gamma}=10 \mathrm{~dB}, M=5, K_{1}=K_{2}=1, r_{P}=1$, $\lambda_{\mathrm{SR}}=\lambda_{\mathrm{RD}}=1, \lambda_{\mathrm{RI}_{11}}=0.5$, and $\lambda_{\mathrm{RI}_{11}}=0.75$. It can be observed from this figure that the channel capacity of the PRS and ORS protocols decreases with the increasing of $\kappa$. Again, we can obverse that the performance of the ORS scheme is better than that of the PRS scheme when the best relay can be selected for the cooperation.

\section{Conclusions}

In this paper, analyzing the impact of hardware impairment and CCI, the end-to-end performance of dual-hop proactive DF relaying networks with $N$ th PRS and $N$ th ORS is investigated. Exact and asymptotic closed-form expressions for the outage probability and average channel capacity of both relay selection schemes were derived. Insightful discussions were provided. For instance, it was shown that, when the system cannot select the best relay for cooperation, the partial relay selection scheme outperforms the opportunistic method under the impact of the same co-channel interference.

\section{ACKNOWLEDGMENTS}

This research is funded by Vietnam National Foundation for Science and Technology Development (NAFOSTED) under grant number 102.01-2014.33.

\section{Appendix A: Proof of TheOrem 1}

Firstly, we rewrite $F_{\psi_{e 2 e}^{\mathrm{PRS}}}(x)$ as follows

$$
F_{\psi_{e 2 e}^{\mathrm{PRS}}}(x)=1-\left(1-F_{\psi_{\mathrm{SR}_{\mathrm{b}}}}(x)\right)\left(1-F_{\psi_{\mathrm{R}_{\mathrm{b}} \mathrm{D}}}(x)\right) .
$$

Thus, in order to attain (A.1), the CDFs $F_{\psi_{\mathrm{SR}_{\mathrm{b}}}}(\cdot)$ and $F_{\psi_{\mathrm{R}_{\mathrm{b}} \mathrm{D}}}(\cdot)$ are required. Considering first the $\mathrm{CDF}$ of $\psi_{\mathrm{SR}_{\mathrm{b}}}$, we have that

$$
\begin{aligned}
F_{\psi_{\mathrm{SR}_{\mathrm{b}}}}(x) & =\operatorname{Pr}\left(\psi_{\mathrm{SR}_{\mathrm{b}}}<x\right) \\
& = \begin{cases}1 ; & \text { if } \mathrm{x} \geq \kappa^{-1} \\
\gamma_{\mathrm{SR}_{\mathrm{b}}}<\frac{x+x Z_{1}}{1-\kappa x} ; & \text { if } \mathrm{x}<\kappa^{-1}\end{cases}
\end{aligned}
$$

For $x<\kappa^{-1}$, (A.2) can be formulated as

$$
F_{\psi_{\mathrm{SR}_{\mathrm{b}}}}(x)=\int_{0}^{+\infty} F_{\gamma_{\mathrm{SR}_{\mathrm{b}}}}\left(\frac{x+x z_{1}}{1-\kappa x}\right) f_{Z_{1}}\left(z_{1}\right) d z_{1} .
$$


Now, using the $N$-best order statistics [30], the CDF of $\gamma_{S}$ can be written as

$$
\begin{aligned}
F_{\gamma_{\mathrm{SR}_{\mathrm{b}}}}(y) & =\sum_{m=1}^{N} C_{M}^{m-1}\left(1-\exp \left(-\Omega_{1} y\right)\right)^{M-m+1} \\
& \times \exp \left(-(m-1) \Omega_{1} y\right) \\
& =1-\sum_{m=1}^{N} \sum_{n=0, n+m>1}^{M-m+1}(-1)^{n+1} C_{M}^{m-1} C_{M-m+1}^{n} \\
& \times \exp \left(-(n+m-1) \Omega_{1} y\right),
\end{aligned}
$$

where $\Omega_{1}=N_{0} \lambda_{\mathrm{SR}} / P$ and $C_{b}^{a}=\frac{b !}{a !(b-a) !}$, with $a$ and $b$ being integers and $b>a$.

Combining (7), (A.3) and (A.4), and after some algebraic manipulation, it follows that

$$
\begin{aligned}
F_{\psi_{\mathrm{SR}_{\mathrm{b}}}}(x) & =1-\sum_{m=1}^{N} \sum_{n=0, m+n>1}^{M-m+1} \sum_{v=1}^{K_{1}}(-1)^{n+1} \Delta_{1} \\
& \times \frac{1-\kappa x}{\Phi_{1}+x} \exp \left(-\frac{\Theta_{1} x}{1-\kappa x}\right),
\end{aligned}
$$

where $\Theta_{1}=(n+m-1) \Omega_{1}, \quad \Delta_{1}=$ $C_{M}^{m-1} C_{M-m+1}^{n} \alpha_{\mathrm{RI}_{1 \mathrm{v}}} /\left(\Theta_{1}-\kappa \Omega_{\mathrm{RI}_{1 \mathrm{v}}}\right), \quad$ and $\Phi_{1}=$ $\Omega_{\mathrm{RI}_{1 \mathrm{v}}} /\left(\Theta_{1}-\kappa \Omega_{\mathrm{RI}_{1 \mathrm{v}}}\right)$. For simplicity, we assume that $\Theta_{1}-\kappa \Omega_{\mathrm{RI}_{1 \mathrm{v}}} \neq 0$.

Similarly, one can see that, if $x \geq \kappa^{-1}, F_{\psi_{\mathrm{R}_{\mathrm{b}} \mathrm{D}}}(x)=1$, while if $x<\kappa^{-1}$, then

$$
F_{\psi_{\mathrm{R}_{\mathrm{b}} \mathrm{D}}}(x)=1-\sum_{t=1}^{K_{2}} \Delta_{2} \frac{1-\kappa x}{\Phi_{2}+x} \exp \left(-\frac{\Omega_{2} x}{1-\kappa x}\right),
$$

where $\Omega_{2}=\lambda_{\mathrm{RD}} N_{0} / P, \Delta_{2}=\alpha_{\mathrm{DI}_{2 \mathrm{t}}} /\left(\Omega_{2}-\Omega_{\mathrm{DI}_{2 \mathrm{t}}} \kappa\right), \Phi_{2}=$ $\Omega_{\mathrm{DI}_{2 \mathrm{t}}} /\left(\Omega_{2}-\kappa \Omega_{\mathrm{DI}_{2 \mathrm{t}}}\right)$, and $\Omega_{2}-\kappa \Omega_{\mathrm{DI}_{2 \mathrm{t}}} \neq 0$.

Finally, by substituting (A.5) and (A.6) into (A.1), (10) is attained, which completes the proof.

\section{APPENDIX B: PROOF OF LEMMA 1}

Without interference sources, (3) and (4) can be rewritten as

$$
\begin{aligned}
\psi_{\mathrm{SR}_{\mathrm{b}}} & =\frac{\gamma_{\mathrm{SR}_{\mathrm{b}}}}{\kappa \gamma_{\mathrm{SR}_{\mathrm{b}}}+1}, \\
\psi_{\mathrm{R}_{\mathrm{b}} \mathrm{D}} & =\frac{\gamma_{\mathrm{R}_{\mathrm{b}} \mathrm{D}}}{\kappa \gamma_{\mathrm{R}_{\mathrm{b}} \mathrm{D}}+1} .
\end{aligned}
$$

Similar to (A.2)-(A.5), the CDFs $F_{\psi_{\mathrm{SR}_{\mathrm{b}}}}(\cdot)$ and $F_{\psi_{\mathrm{R}_{\mathrm{b}} \mathrm{D}}}(\cdot)$ can be obtained as

$$
\begin{aligned}
F_{\psi_{\mathrm{SR}_{\mathrm{b}}}}(x) & =1-\sum_{m=1}^{N} \sum_{n=0, m+n \neq 1}^{M-m+1}(-1)^{n+1} \\
& \times C_{M}^{m-1} C_{M-m+1}^{n} \exp \left(-\frac{\Theta_{1} x}{1-\kappa x}\right), \\
F_{\psi_{\mathrm{R}_{\mathrm{b}} \mathrm{D}}}(x) & =1-\exp \left(-\frac{\Omega_{2} x}{1-\kappa x}\right) .
\end{aligned}
$$

Then, combining the above results with (A.1), the proof of Lemma 1 is concluded.

\section{APPENDIX C: PROOF OF LEMMA 2}

From (A.1) in Appendix A, we can approximate $F_{\psi_{\mathrm{e} 2 \mathrm{e}}^{\mathrm{PRS}}}(x)$ at high SNR region by

$$
F_{\psi_{\mathrm{e} 2 \mathrm{e}}^{\mathrm{PRS}}}(x) \stackrel{\bar{\gamma} \rightarrow+\infty}{\approx} F_{\psi_{\mathrm{SR}_{\mathrm{b}}}}(x)+F_{\psi_{\mathrm{R}_{\mathrm{b}} \mathrm{D}}}(x),
$$

where $\psi_{\mathrm{SR}_{\mathrm{b}}}$ and $\psi_{\mathrm{R}_{\mathrm{b}} \mathrm{D}}$ are given as (B.1) in Appendix B.

In addition, since $1-\exp (-t) \stackrel{t \rightarrow 0}{\overbrace{}^{*}} t$ and $\exp (-t) \stackrel{t \rightarrow 0}{\overbrace{}^{\circ}} 1$, asymptotic expressions for (B.2) can be written as

$$
\begin{aligned}
& F_{\gamma_{1 b}}(x) \stackrel{\bar{\gamma} \rightarrow+\infty}{\approx} \sum_{m=1}^{N} C_{M}^{m-1}\left(\frac{\Omega_{1} x}{1-\kappa x}\right)^{M-m+1} \\
& \stackrel{\bar{\gamma} \rightarrow+\infty}{\approx} C_{M}^{N-1}\left(\frac{\Omega_{1} x}{1-\kappa x}\right)^{M-N+1}, \\
& F_{\gamma_{2 b}}(x) \stackrel{\gamma \rightarrow+\infty}{\approx} \frac{\Omega_{2} x}{1-\kappa x} .
\end{aligned}
$$

Combining (C.1) and (C.2), (14) is attained, which completes the proof.

\section{APPENDIX D: PROOF OF THEOREM 3}

Firstly, it is easy to see that $F_{\psi_{e 2}^{\mathrm{ORS}}(x)}=1$ when $x \geq \kappa^{-1}$. Thus, considering the case when $x<\kappa^{-1}$, (16) can be rewritten as

$$
\begin{aligned}
& F_{\psi_{e 2 e}^{\mathrm{ORS}}}(x)=1-\operatorname{Pr}\left(\psi_{\mathrm{SR}_{\mathrm{b}}} \geq x, \psi_{\mathrm{R}_{\mathrm{b}} \mathrm{D}} \geq x\right) \\
& =1-\operatorname{Pr}\left(\gamma_{\mathrm{SR}_{\mathrm{b}}} \geq \frac{x+x Z_{1}}{1-\kappa x}, \gamma_{\mathrm{R}_{\mathrm{b}} \mathrm{D}} \geq \frac{x+x Z_{2}}{1-\kappa x}\right) .
\end{aligned}
$$

Since $\gamma_{\mathrm{SR}_{\mathrm{b}}}$ and $\gamma_{\mathrm{R}_{\mathrm{b}} \mathrm{D}}$ are not independent, the method proposed in [11] will be employed to calculate (D.1). Initially, we will derive the probability $\operatorname{Pr}\left(\gamma_{\mathrm{SR}_{\mathrm{b}}} \geq u_{1}, \gamma_{\mathrm{R}_{\mathrm{b}} \mathrm{D}} \geq u_{2}\right)$. To this end, similar to [11], this probability can be formulated as

$$
\operatorname{Pr}\left(\gamma_{\mathrm{SR}_{\mathrm{b}}} \geq u_{1}, \gamma_{\mathrm{R}_{\mathrm{b}} \mathrm{D}} \geq u_{2}\right)=\int_{0}^{+\infty} \frac{\partial G(z)}{\partial z} \frac{f_{T_{\max }}(z)}{f_{T_{i}}(z)} d z .
$$

In (D.2), $T_{\max }=N$ th $\max _{m=1,2, \ldots, M} \min \left(\gamma_{\mathrm{SR}_{\mathrm{m}}}, \gamma_{\mathrm{R}_{\mathrm{m}} \mathrm{D}}\right)$, in which its CDF can be expressed similarly to (A.4) as

$$
\begin{aligned}
F_{T_{\max }}(z) & =1-\sum_{m=1}^{N} \sum_{n=0, n+m>1}^{M-m+1}(-1)^{n+1} C_{M}^{m-1} C_{M-m+1}^{n} \\
& \times \exp (-(n+m-1) \Omega z),
\end{aligned}
$$

where $\Omega=\Omega_{1}+\Omega_{2}$. Thus, the PDF of $T_{\max }$ can be derived as

$$
\begin{aligned}
f_{T_{\max }}(z) & =\sum_{m=1}^{N} \sum_{n=0, m+n>1}^{M-m+1}(-1)^{n} C_{M}^{m-1} C_{M-m+1}^{n} \\
& \times(m+n-1) \Omega \exp (-(n+m-1) \Omega z) .
\end{aligned}
$$

By its turn, in (D.2), $T_{i}=\min \left(\gamma_{\mathrm{SR}_{\mathrm{i}}}, \gamma_{\mathrm{R}_{\mathrm{i}} \mathrm{D}}\right), i=1,2, \ldots, M$, such that its PDF can be expressed as

$$
f_{T_{i}}(z)=\Omega \exp (-\Omega z) .
$$

Finally, the term $G(z)$ in (D.2) can be formulated as

$$
G(z)=\operatorname{Pr}\left(\gamma_{\mathrm{SR}_{\mathrm{i}}} \geq u_{1}, \gamma_{\mathrm{R}_{\mathrm{i}} \mathrm{D}} \geq u_{2}, \min \left(\gamma_{\mathrm{SR}_{\mathrm{i}}}, \gamma_{\mathrm{R}_{\mathrm{i}} \mathrm{D}}\right)<z\right) .
$$


In order to calculate $G(z)$, two cases will be considered:

- Case 1: $u_{1} \geq u_{2}$

In this case, $G(z)$ can be obtained as

$$
G(z)= \begin{cases}0 ; & \text { if } z \leq u_{2} \\ \exp \left(-\Omega_{1} u_{1}-\Omega_{2} u_{2}\right) & \\ -\exp \left(-\Omega_{1} u_{1}-\Omega_{2} z\right) ; & \text { if } u_{2} \leq z<u_{1} \\ \exp \left(-\Omega_{1} u_{1}-\Omega_{2} u_{2}\right) & \\ -\exp (-\Omega z) ; & \text { if } z \geq u_{1}\end{cases}
$$

- Case 2: $u_{1}<u_{2}$

In this case, it follows that

$$
G(z)= \begin{cases}0 ; & \text { if } z \leq u_{1} \\ \exp \left(-\Omega_{1} u_{1}-\Omega_{2} u_{2}\right) & \text { if } u_{2} \leq z<u_{1} \\ -\exp \left(-\Omega_{2} u_{2}-\Omega_{1} z\right) ; & \text { if } z \geq u_{1}\end{cases}
$$

Combining (D.4), (D.5), (D.7) and (D.8), and after some algebraic manipulations, $\operatorname{Pr}\left(\gamma_{\mathrm{SR}_{\mathrm{b}}} \geq u_{1}, \gamma_{\mathrm{R}_{\mathrm{b}} \mathrm{D}} \geq u_{2}\right)$ is derived for Case 1 and Case 2 in (D.9) and (D.10), respectively, shown at the top of next page.

Now, replacing $u_{1}=\left(x+x Z_{1}\right) /(1-\kappa x)$ and $u_{2}=$ $\left(x+x Z_{2}\right) /(1-\kappa x)$ in (D.9) and (D.10), the outage probability $F_{\psi_{e 2 e}^{\mathrm{ORS}}}(x)$ can be calculated as ${ }^{5}$

$$
F_{\psi_{e 2 e}^{\mathrm{ORS}}}(x)=1-S_{1}-S_{2},
$$

where

$$
\begin{aligned}
S_{1}= & \int_{0}^{+\infty} \int_{0}^{z_{1}} \operatorname{Pr}\left(\gamma_{\mathrm{SR}_{\mathrm{b}}} \geq \frac{x+x z_{1}}{1-\kappa x}, \gamma_{\mathrm{R}_{\mathrm{b}} \mathrm{D}} \geq \frac{x+x z_{2}}{1-\kappa x}\right) \\
& f_{Z_{1}}\left(z_{1}\right) f_{Z_{2}}\left(z_{2}\right) d z_{2} d z_{1}, \\
S_{2}= & \int_{0}^{+\infty} \int_{0}^{z_{2}} \operatorname{Pr}\left(\gamma_{\mathrm{SR}_{\mathrm{b}}} \geq \frac{x+x z_{1}}{1-\kappa x}, \gamma_{\mathrm{R}_{\mathrm{b}} \mathrm{D}} \geq \frac{x+x z_{2}}{1-\kappa x}\right) \\
& f_{Z_{1}}\left(z_{1}\right) f_{Z_{2}}\left(z_{2}\right) d z_{1} d z_{2} .
\end{aligned}
$$

By substituting (7) and (D.9) into (D.12), and after some algebraic manipulations, it follows that

$$
\begin{aligned}
S_{1} & =\sum_{m=1}^{N} \sum_{n=0, m+n>1}^{M-m+1} \sum_{v=1}^{K_{1}} \sum_{t=1}^{K_{2}}(-1)^{n+1} \\
& \times\left[\frac{\Delta_{3}(1-\kappa x)^{2}}{\left(\Phi_{3}+x\right)\left(\Phi_{5}+x\right)}+\frac{\Delta_{4}(1-\kappa x)^{2}}{\left(\Phi_{4}+x\right)\left(\Phi_{5}+x\right)}\right] \\
& \times \exp \left(-\frac{\Theta_{2} x}{1-\kappa x}\right),
\end{aligned}
$$

where $\Theta_{2}=(n+m-1) \Omega, \quad \Phi_{3}=$ $\Omega_{\mathrm{RI}_{1 \mathrm{v}}} /\left(\Omega_{1}-\kappa \Omega_{\mathrm{RI}_{1 \mathrm{v}}}\right), \quad \Phi_{4}=\Omega_{\mathrm{RI}_{1 \mathrm{v}}} /\left(\Theta_{2}-\kappa \Omega_{\mathrm{RI}_{1 \mathrm{v}}}\right)$, $\Phi_{5}=\left(\Omega_{\mathrm{RI}_{1 \mathrm{v}}}+\Omega_{\mathrm{DI}_{2 \mathrm{t}}}\right) /\left(\Theta_{2}-\kappa\left(\Omega_{\mathrm{RI}_{1 \mathrm{v}}}+\Omega_{\mathrm{DI}_{2 \mathrm{t}}}\right)\right)$, and

$$
\begin{aligned}
\Delta_{3} & =(n+m-1) C_{M}^{m-1} C_{M-m+1}^{n} \frac{\Omega_{2} \alpha_{\mathrm{RI}_{1 \mathrm{v}}} \alpha_{\mathrm{DI}_{2 \mathrm{t}}}}{\Omega_{2}+(n+m-2) \Omega} \\
& \times \frac{1}{\left(\Theta_{2}-\kappa\left(\Omega_{\mathrm{RI}_{1 \mathrm{v}}}+\Omega_{\mathrm{DI}_{2 \mathrm{t}}}\right)\right)\left(\Omega_{1}-\kappa \Omega_{\mathrm{RI}_{1 \mathrm{v}}}\right)}, \\
\Delta_{4} & =(n+m-2) C_{M}^{m-1} C_{M-m+1}^{n} \frac{\Omega_{1} \alpha_{\mathrm{RI}_{1 \mathrm{v}}} \alpha_{\mathrm{DI}_{2 \mathrm{t}}}}{\Omega_{2}+(n+m-2) \Omega} \\
& \times \frac{1}{\left(\Theta_{2}-\kappa \Omega_{\mathrm{RI}_{1 \mathrm{v}}}\right)\left(\Theta_{2}-\kappa\left(\Omega_{\mathrm{RI}_{1 \mathrm{v}}}+\Omega_{\mathrm{DI}_{2 \mathrm{t}}}\right)\right)} .
\end{aligned}
$$

${ }^{5} u_{1} \geq u_{2}$ is equivalent to $Z_{1} \geq Z_{2}$, and vice versa.
Similarly, from (7), (D.10) and (D.13), $S_{2}$ can be obtained as

$$
\begin{aligned}
S_{2} & =\sum_{m=1}^{N} \sum_{n=0, m+n>1}^{M-m+1} \sum_{v=1}^{K_{1}} \sum_{t=1}^{K_{2}}(-1)^{n+1} \\
& \times\left[\frac{\Delta_{5}(1-\kappa x)^{2}}{\left(\Phi_{6}+x\right)\left(\Phi_{5}+x\right)}+\frac{\Delta_{6}(1-\kappa x)^{2}}{\left(\Phi_{7}+x\right)\left(\Phi_{5}+x\right)}\right] \\
& \times \exp \left(-\frac{\Theta_{2} x}{1-\kappa x}\right),
\end{aligned}
$$

where $\Phi_{6}=\Omega_{\mathrm{DI}_{2 \mathrm{t}}} /\left(\Omega_{2}-\kappa \Omega_{\mathrm{DI}_{2 \mathrm{t}}}\right), \quad \Phi_{7}=$ $\Omega_{\mathrm{DI}_{2 \mathrm{t}}} /\left(\Theta_{2}-\kappa \Omega_{\mathrm{DI}_{2 \mathrm{t}}}\right)$, and

$$
\begin{aligned}
\Delta_{5} & =(n+m-1) C_{M}^{m-1} C_{M-m+1}^{n} \frac{\Omega_{1} \alpha_{\mathrm{RI}_{1 \mathrm{v}}} \alpha_{\mathrm{DI}_{2 \mathrm{t}}}}{\Omega_{1}+(n+m-2) \Omega} \\
& \times \frac{1}{\left(\Theta_{2}-\kappa\left(\Omega_{\mathrm{RI}_{1 \mathrm{v}}}+\Omega_{\mathrm{DI}_{2 \mathrm{t}}}\right)\right)\left(\Omega_{2}-\kappa \Omega_{\mathrm{DI}_{2 \mathrm{t}}}\right)}, \\
\Delta_{6} & =(n+m-2) C_{M}^{m-1} C_{M-m+1}^{n} \frac{\Omega_{2} \alpha_{\mathrm{RI}_{1 \mathrm{v}}} \alpha_{\mathrm{DI}_{2 \mathrm{t}}}}{\Omega_{1}+(n+m-2) \Omega} \\
& \times \frac{1}{\left(\Theta_{2}-\kappa \Omega_{\mathrm{DI}_{2 \mathrm{t}}}\right)\left(\Theta_{2}-\kappa\left(\Omega_{\mathrm{RI}_{1 \mathrm{v}}}+\Omega_{\mathrm{DI}_{2 \mathrm{t}}}\right)\right)} .
\end{aligned}
$$

Finally, combining (D.11), (D.14) and (D.15), the proof is concluded.

\section{Appendix E: Proof of Theorem 5}

Firstly, we rewrite (10) as

$$
\begin{aligned}
F_{\psi_{e 2 e}^{\mathrm{PRS}}}(x) & =1-\sum_{m=1}^{N} \sum_{n=0, m+n>1}^{M-m+1} \sum_{v=1}^{K_{1}} \sum_{t=1}^{K_{2}}(-1)^{n+1} \Delta_{1} \Delta_{2} \\
& \times\left(\frac{\delta_{1}}{\Phi_{1}+x}+\frac{\delta_{2}}{\Phi_{2}+x}+\kappa^{2}\right) \\
& \times \exp \left(-\frac{\left(\Theta_{1}+\Omega_{2}\right) x}{1-\kappa x}\right)
\end{aligned}
$$

where $\delta_{1}=\left(1+\kappa \Phi_{1}\right)^{2} /\left(\Phi_{2}-\Phi_{1}\right)$ and $\delta_{2}=$ $\left(1+\kappa \Phi_{2}\right)^{2} /\left(\Phi_{1}-\Phi_{2}\right)$. Now, by substituting (E.1) into (21), we have

$$
\begin{aligned}
& C_{\text {avg }}^{\mathrm{PRS}}=\frac{1}{2 \ln 2} \sum_{m=1}^{N} \sum_{n=0, m+n>1}^{M-m+1} \sum_{v=1}^{K_{1}} \sum_{t=1}^{K_{2}}(-1)^{n+1} \Delta_{1} \Delta_{2} \\
& \times \int_{0}^{\kappa^{-1}}\left(\frac{\delta_{1}}{(1+x)\left(\Phi_{1}+x\right)}+\frac{\delta_{2}}{(1+x)\left(\Phi_{2}+x\right)}+\frac{\kappa^{2}}{1+x}\right) \\
& \times \exp \left(-\frac{\left(\Theta_{1}+\Omega_{2}\right) x}{1-\kappa x}\right) d x .
\end{aligned}
$$

Next, rewriting (E.2) as

$$
\begin{aligned}
C_{\mathrm{avg}}^{\mathrm{PRS}} & =\frac{1}{2 \ln 2} \sum_{m=1}^{N} \sum_{n=0, m+n>1}^{M-m+1} \sum_{v=1}^{K_{1}} \sum_{t=1}^{K_{2}}(-1)^{n+1} \Delta_{1} \Delta_{2} \\
& \times \int_{0}^{\kappa^{-1}} L_{1}(x) \exp \left(-\frac{\left(\Theta_{1}+\Omega_{2}\right) x}{1-\kappa x}\right) d x,
\end{aligned}
$$

in which

$$
\begin{aligned}
L_{1}(x) & =\frac{\delta_{1}}{\Phi_{1}-1}\left(\frac{1}{1+x}-\frac{1}{\Phi_{1}+x}\right) \\
& +\frac{\delta_{2}}{\Phi_{2}-1}\left(\frac{1}{1+x}-\frac{1}{\Phi_{2}+x}\right)+\frac{\kappa^{2}}{1+x} .
\end{aligned}
$$




$$
\begin{aligned}
& \operatorname{Pr}\left(\gamma_{\mathrm{SR}_{\mathrm{b}}} \geq u_{1}, \gamma_{\mathrm{R}_{\mathrm{b}} \mathrm{D}} \geq u_{2}\right)=\sum_{m=1}^{N} \sum_{n=0, m+n>1}^{M-m+1}(-1)^{n} C_{M}^{m-1} C_{M-m+1}^{n} \\
& \times\left[\frac{(n+m-1) \Omega_{2}}{\Omega_{2}+(n+m-2) \Omega} \exp \left(-\Omega_{1} u_{1}-\left(\Omega_{2}+(n+m-2) \Omega\right) u_{2}\right)+\frac{(n+m-2) \Omega_{1}}{\Omega_{2}+(n+m-2) \Omega} \exp \left(-(n+m-1) \Omega u_{1}\right)\right]
\end{aligned}
$$

$$
\begin{aligned}
& \operatorname{Pr}\left(\gamma_{\mathrm{SR}_{\mathrm{b}}} \geq u_{1}, \gamma_{\mathrm{R}_{\mathrm{b}} \mathrm{D}} \geq u_{2}\right)=\sum_{m=1}^{N} \sum_{n=0, m+n>1}^{M-m+1}(-1)^{n} C_{M}^{m-1} C_{M-m+1}^{n} \\
& \times\left[\frac{(n+m-1) \Omega_{1}}{\Omega_{1}+(n+m-2) \Omega} \exp \left(-\Omega_{2} u_{2}-\left(\Omega_{1}+(n+m-2) \Omega\right) u_{1}\right)+\frac{(n+m-2) \Omega_{2}}{\Omega_{1}+(n+m-2) \Omega} \exp \left(-(n+m-1) \Omega u_{2}\right)\right]
\end{aligned}
$$

Finally, applying (23) for the corresponding integral in (E.3), we finish the proof of Theorem 5 .

\section{APpendix F: Proof of TheOREM 7}

Firstly, we rewrite (16) as (F.1), shown at the top of this page, where $\delta_{3}=\left(1+\kappa \Phi_{3}\right)^{2} /\left(\Phi_{5}-\Phi_{3}\right), \delta_{4}=$ $\left(1+\kappa \Phi_{5}\right)^{2} /\left(\Phi_{3}-\Phi_{5}\right), \delta_{5}=\left(1+\kappa \Phi_{4}\right)^{2} /\left(\Phi_{5}-\Phi_{4}\right), \delta_{6}=$ $\left(1+\kappa \Phi_{5}\right)^{2} /\left(\Phi_{4}-\Phi_{5}\right), \delta_{7}=\left(1+\kappa \Phi_{6}\right)^{2} /\left(\Phi_{5}-\Phi_{6}\right), \delta_{8}=$ $\left(1+\kappa \Phi_{5}\right)^{2} /\left(\Phi_{6}-\Phi_{5}\right), \delta_{9}=\left(1+\kappa \Phi_{7}\right)^{2} /\left(\Phi_{5}-\Phi_{7}\right)$, and $\delta_{10}=\left(1+\kappa \Phi_{5}\right)^{2} /\left(\Phi_{7}-\Phi_{5}\right)$. Now, by substituting (F.1) into (21), and after some algebraic manipulations, it follows that

$$
\begin{aligned}
C_{\text {avg }}^{\mathrm{ORS}} & =\frac{1}{2 \ln 2} \sum_{m=1}^{N} \sum_{n=0, m+n>1}^{M-m+1} \sum_{v=1}^{K_{1}} \sum_{t=1}^{K_{2}}(-1)^{n+1} \\
& \times \int_{0}^{\kappa^{-1}} L_{2}(x) \exp \left(-\frac{\Theta_{2} x}{1-\kappa x}\right) d x,
\end{aligned}
$$

where $L_{2}(x)$ is a function of $x$, which is given in (F.3), shown at the top of next page. Next, applying (23) for the corresponding integral in (F.2), the proof is concluded.

\section{REFERENCES}

[1] J. N. Laneman, D. N. C. Tse, and G. W. Wornell, "Cooperative diversity in wireless networks: Efficient protocols and outage behavior," vol. 50, no. 12, pp. 3062-3080, Dec. 2004.

[2] A. Bletsas, A. Khisti, D. P. Reed, and A. Lippman, "A simple cooperative diversity method based on network path selection," vol. 24, no. 3, pp. 559-572, Mar. 2006.

[3] Y. Zhao, R. Adve, and T. J. Lim, "Symbol error rate of selection amplifyand-forward relay systems," IEEE Commun. Lett., vol. 10, no. 11, pp. 757-759, Nov. 2006.

[4] A. Bletsas, H. Shin, and M. Z. Win, "Outage optimality of opportunistic amplify-and-forward relaying," IEEE Commun. Lett., vol. 11, no. 3, pp. 261-263, Mar. 2007.

[5] E. Beres and R. Adve, "Selection cooperation in multi-source cooperative networks," vol. 7, no. 1, pp. 118-127, Jan. 2008.

[6] D. S. Michalopoulos and G. K. Karagiannidis, "Performance analysis of single relay selection in Rayleigh fading," vol. 7, no. 10, pp. 3718-3724, Oct. 2008.

[7] B. Maham and A. Hjorungnes, "Performance analysis of amplify-andforward opportunistic relaying in Rician fading," vol. 16, no. 8, pp. 643-646, Aug. 2009.

[8] F. Xu, F. C. M. Lau, Q. F. Zhou, and D.-W. Yue, "Outage performance of cooperative communication systems using opportunistic relaying and selection combining receiver," vol. 16, pp. 237-240, Apr. 2009.

[9] T. Q. Duong, V. N. Q. Bao, and H.-J. Zepernick, "On the performance of selection decode-and-forward relay networks over Nakagami- $m$ fading channels," vol. 13, no. 8, pp. 172-174, Mar. 2009.
[10] A. Adinoyi, Y. Fan, H. Yanikomeroglu, H. V. Poor, and F. Al-Shaalan, "Performance of selection relaying and cooperative diversity," vol. 8, no. 12, pp. 5790-5795, Dec. 2009.

[11] K. Tourki, H.-C. Yang, and M.-S. Alouini, "Accurate outage analysis of incremental decode-and-forward opportunistic relaying," vol. 10, no. 4, pp. 1021-1025, Apr. 2011.

[12] I. Krikidis, J. Thompson, S. McLaughlin, and N. Goertz, "Amplify-andforward with partial relay selection," IEEE Commun. Lett., vol. 12, no. 4, pp. 235-237, Apr. 2008.

[13] D. B. da Costa and S. Aïssa, "Capacity analysis of cooperative systems with relay selection in Nakagami- $m$ fading," vol. 13, no. 9, pp. 637-639, Sep. 2009.

[14] K. Yan, J. Jiang, Y. G. Wang, and H. T. Liu, "Outage probability of selection cooperation with MRC in Nakagami- $m$ fading channels," vol. 16, no. 12, pp. 1031-1034, Dec. 2009.

[15] D. B. da Costa and S. Aissa, "End-to-end performance of dual-hop semi-blind relaying systems with partial relay selection," vol. 8 , no. 8, pp. 4306-4315, Aug. 2009.

[16] G. C. Alexandropoulos, A. Papadogiannis, and K. Berberidis, "Performance analysis of cooperative networks with relay selection over Nakagami- $m$ fading channels," vol. 17, no. 5, pp. 1-4, May 2010.

[17] H. Ding, J. Ge, D. B. da Costa, and Z. Jiang, "Diversity and coding gains of fixed-gain amplify-and-forward with partial relay selection in Nakagami- $m$ fading," vol. 14, no. 8, pp. 734-736, Aug. 2010.

[18] H. Suraweera, M. Soysa, C. Tellambura, and H. Garg, "Performance analysis of partial relay selection with feedback delay," vol. 17, no. 6, pp. 531-534, June 2010.

[19] Y. Chen, C.-X. Wang, H. Xiao, and D. Yuan, "Novel partial selection schemes for AF relaying in Nakagami- $m$ fading channels," vol. 60 , no. 7, pp. 3497-3503, Sept. 2011.

[20] E. Costa and S. Pupolin, " $m$-QAM-OFDM system performance in the presence of a nonlinear amplifier and phase noise," IEEE Trans. Commun., vol. 50, no. 3, pp. 462-472, Mar. 2002.

[21] M. Mokhtar, A. Gomaa, and N. Al-Dhahir, "OFDM AF relaying under I/Q imbalance: Performance analysis and baseband compensation," vol. 61, no. 4, pp. 1304-1313, Apr. 2013.

[22] M. Mokhtar, A.-A. A. Boulogeorgos, G. K. Karagiannidis, and N. AlDhahir, "OFDM opportunistic relaying under joint transmit/receive I/Q imbalance," vol. 62, no. 5, pp. 1458-1468, May 2014

[23] E. Björnson, M. Matthaiou, and M. Debbah, "A new look at dualhop relaying: Performance limits with hardware impairments," vol. 61, no. 11, pp. 4512-4525, Nov. 2013.

[24] M. Matthaiou and A. Papadogiannis, "Two-way relaying under the presence of relay transceiver hardware impairments," vol. 17 , no. 6 , pp. 1136 - 1139, Jun. 2013.

[25] A. Salhab, F. Al-Qahtani, S. Zummo, and H. Alnuweiri, "Exact outage probability of opportunistic DF relay systems with interference at both the relay and the destination over Nakagami- $m$ fading channels," vol. 62 , no. 2, pp. 920 - 927, Feb. 2013.

[26] K. T. Hemachandra and N. C. Beaulieu, "Outage analysis of opportunistic scheduling in dual-hop multiuser relay networks in the presence of interference," vol. 61, no. 5, pp. 1786 - 1796, May 2013.

[27] E. Soleimani-Nasab, M. Matthaiou, M. Ardebilipour, and G. Karagiannidis, "Two-way AF relaying in the presence of co-channel interference," vol. 61 , no. 8 , pp. 3156 - 3169, Aug. 2013. 


$$
\begin{aligned}
F_{\psi_{e 2 e}^{\mathrm{ORS}}} & =1-\sum_{m=1}^{N} \sum_{n=0, m+n>1}^{M-m+1} \sum_{v=1}^{K_{1}} \sum_{t=1}^{K_{2}}(-1)^{n+1} \exp \left(-\frac{\Theta_{2} x}{1-\kappa x}\right) \\
& \times \Delta_{3}\left(\frac{\delta_{3}}{\Phi_{3}+x}+\frac{\delta_{4}}{\Phi_{5}+x}+\kappa^{2}\right)+\Delta_{4}\left(\frac{\delta_{5}}{\Phi_{4}+x}+\frac{\delta_{6}}{\Phi_{5}+x}+\kappa^{2}\right)+\Delta_{5}\left(\frac{\delta_{7}}{\Phi_{6}+x}+\frac{\delta_{8}}{\Phi_{5}+x}+\kappa^{2}\right) \\
& +\Delta_{6}\left(\frac{\delta_{9}}{\Phi_{7}+x}+\frac{\delta_{10}}{\Phi_{5}+x}+\kappa^{2}\right) .
\end{aligned}
$$

$$
\begin{aligned}
L_{2}(x) & =\Delta_{3}\left(\frac{\delta_{3}}{\Phi_{3}-1}\left(\frac{1}{1+x}-\frac{1}{\Phi_{3}+x}\right)+\frac{\delta_{4}}{\Phi_{5}-1}\left(\frac{1}{1+x}-\frac{1}{\Phi_{5}+x}\right)+\frac{\kappa^{2}}{1+x}\right) \\
& +\Delta_{4}\left(\frac{\delta_{5}}{\Phi_{4}-1}\left(\frac{1}{1+x}-\frac{1}{\Phi_{4}+x}\right)+\frac{\delta_{6}}{\Phi_{5}-1}\left(\frac{1}{1+x}-\frac{1}{\Phi_{5}+x}\right)+\frac{\kappa^{2}}{1+x}\right) \\
& +\Delta_{5}\left(\frac{\delta_{7}}{\Phi_{6}-1}\left(\frac{1}{1+x}-\frac{1}{\Phi_{6}+x}\right)+\frac{\delta_{8}}{\Phi_{5}-1}\left(\frac{1}{1+x}-\frac{1}{\Phi_{5}+x}\right)+\frac{\kappa^{2}}{1+x}\right) \\
& +\Delta_{6}\left(\frac{\delta_{9}}{\Phi_{7}-1}\left(\frac{1}{1+x}-\frac{1}{\Phi_{7}+x}\right)+\frac{\delta_{10}}{\Phi_{5}-1}\left(\frac{1}{1+x}-\frac{1}{\Phi_{5}+x}\right)+\frac{\kappa^{2}}{1+x}\right) .
\end{aligned}
$$

[28] A. Bletsas, H. Shin, and M. Z. Win, "Cooperative communications with outage-optimal opportunistic relaying," vol. 6, no. 9, pp. 3450-3460, Sept. 2007.

[29] I. S. Gradshteyn and I. M. Ryzhik, Table of Integrals, Series, and Products, 6th ed. San Diego, CA: Academic, 2000.

[30] S. Ikki and M. Ahmed, "On the performance of adaptive decode-andforward cooperative diversity with the nth best-relay selection scheme," in Proc. Global Telecommunications Conference, Honolulu, HI, Nov.Dec. 2009.

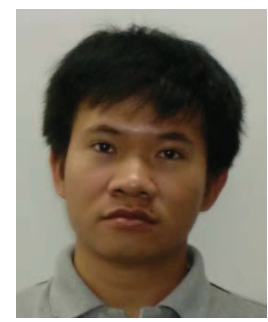

Tran Trung Duy was born in Nha Trang city, Vietnam, in 1984. He received the B.E. degree in Electronics and Telecommunications Engineering from the French-Vietnamese training program for excellent engineers (PFIEV), Ho Chi Minh City University of Technology, Vietnam in 2007. In 2013, he received the Ph.D degree in electrical engineering from University of Ulsan, South Korea. In 2013, he joined the Department of Telecommunications, Posts and Telecommunications Institute of Technology (PTIT), as a lecturer. His major research interests are cooperative communications, cognitive radio, and physical layer security.

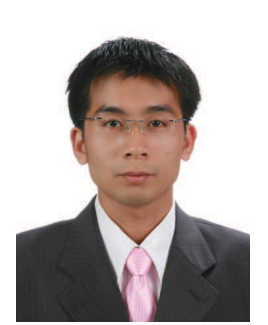

Trung Q. Duong (S'05, M'12, SM'13) received his $\mathrm{Ph} . \mathrm{D}$. degree in Telecommunications Systems from Blekinge Institute of Technology (BTH), Sweden in 2012, and then continued working at BTH as a project manager. Since 2013, he has joined Queen's University Belfast, UK as a Lecturer (Assistant Professor). He held a visiting position at Polytechnic Institute of New York University and Singapore University of Technology and Design in 2009 and 2011, respectively. His current research interests include cooperative communications, cognitive radio networks, physical layer security, massive MIMO, cross-layer design, mmwaves communications, and localization for radios and networks.

Dr. Duong has served as an Editor for the IEEE COMMUNICATIONS LETTERS, IET COMMUNICATIONS, WILEY TRANSACTIONS ON EMERGING Telecommunications Technologies. He has also served as the Lead Guest Editor of the special issue on "Location Awareness for Radios and Networks" of the IEEE JOURnal IN SELECTED AREAS ON COMMUNICATIONS, the Lead Guest Editor of the special issue on "Secure Physical Layer Communications" of the IET COMMUNiCATIONS, Guest Editor of the special issue on "Green Media: The Future of Wireless Multimedia Networks" of the IEEE Wireless Communications Magazine, Guest Editor of the special issue on "Millimeter Wave Communications for 5G" and "Energy Harvesting Communications" of the IEEE COMMUNICATIONS MAGAZINE, Guest Editor of the special issue on "Cooperative Cognitive Networks" of the EURASIP JOURNAL ON WIRELESS COMMUNICATIONS AND NETWORKING, Guest Editor of special issue on "Security Challenges and Issues in Cognitive Radio Networks" of the EURASIP JOURNAL ON Advances Signal Processing. He was awarded the Best Paper Award at the IEEE Vehicular Technology Conference (VTC-Spring) in 2013, IEEE International Conference on Communications (ICC) 2014, and the Exemplary Reviewer Certificate of the IEEE Communications Letters in 2012. 


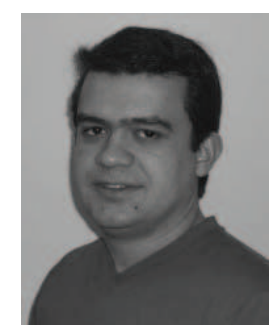

Daniel Benevides da Costa was born in Fortaleza, Ceará, Brazil, in 1981. He received the B.Sc. degree in telecommunications from the Military Institute of Engineering, Rio de Janeiro, Brazil, in 2003 and the M.Sc. and Ph.D. degrees in telecommunications from the University of Campinas, Campinas, Brazil, in 2006 and 2008, respectively. His Ph.D. dissertation was awarded the Best Ph.D. Thesis in Electrical Engineering by the Brazilian Ministry of Education (CAPES) at the 2009 CAPES Thesis Contest. From 2008 to 2009, he was a Postdoctoral Research Fellow with INRS-EMT, University of Quebec, Montreal, QC, Canada. At that time, he was the recipient of two scholarships: 1) the Merit Scholarship Program for Foreign Students in Quebec and 2) the Natural Sciences and Engineering Research Council of Canada Postdoctoral Scholarship. Since 2010, he has been with the Federal University of Ceará, Brazil, where he is currently an Assistant Professor.

Prof. da Costa has authored or coauthored more than 60 papers in IEEE/IET journals and more than 45 papers in international conferences. His research interests lie in the area of wireless communications and include channel modeling and characterization, relaying/multihop/mesh networks, cooperative systems, cognitive radio networks, tensor modeling, physical layer security, and performance analysis/design of multiple-input multiple-output systems $\mathrm{He}$ is currently an Editor of the IEEE COMMUNICATIONS LETTERS, the IEEE TRANSACTIONS ON VEHICULAR TECHNOLOGY, the EURASIP JOURNAL ON WIRELESS COMMUNICATIONS AND NETWORKING, and the KSII TRANSACTIONS ON INTERNET AND INFORMATION SYSTEMS. He has also served as Associate Technical Editor for the IEEE COMMUNICATIONS MAGAZINE. He served as the Lead Guest Editor for EURASIP JOURNAL ON WIRELESS COMMUNICATIONS AND NETWORKING in the Special Issue on "Cooperative Cognitive Networks", Lead Guest Editor for KSII TRANSACTIONS ON INTERNET AND INFORMATION SYSTEMS in the Special Issue on "Cognitive Radio Networks: Survey, Tutorial, and New Introduction", and a Guest Editor for IET COMMUNICATIONS in the Special Issue on "Secure Physical Layer Communications". Also, he was a Workshop Chair of the 2nd International Conference on Computing, Management and Telecommunications (ComManTel 2014) and he served as a TPC chair for the IEEE GLOBECOM 2013, Workshop on Trusted Communications with Physical Layer Security. He also acts as a reviewer for major international journals of the IEEE and IET, and he has been Member of the Technical Program Committee of several international conferences, such as ICC, WCNC, GLOBECOM, PIRMC, and VTC. He is currently a Scientific Consultant of the National Council of Scientific and Technological Development (CNPq), Brazil, and of the Brazilian Ministry of Education (CAPES). He is also a Productivity Research Fellow of CNPq. From 2010 to 2012, he was a Productivity Research Fellow of the Ceará Council of Scientific and Technological Development (FUNCAP). Currently, he is a member of the Advisory Board of FUNCAP, Area: Telecommunications. He is also the recipient of three conference paper awards: one at the 2009 IEEE International Symposium on Computers and Communications, one at the 13th International Symposium on Wireless Personal Multimedia Communications in 2010, and another at the XXIX Brazilian Telecommunications Symposium in 2011. In 2013, he received the Exemplary Reviewer Certificate of the IEEE Wireless Communications Letters and the Certificate of Appreciation of Top Associate Editor for outstanding contributions to IEEE Transactions on Vehicular Technology. He is Senior Member of IEEE, Member of IEEE Communications Society, IEEE Vehicular Technology Society, and Brazilian Telecommunications Society.

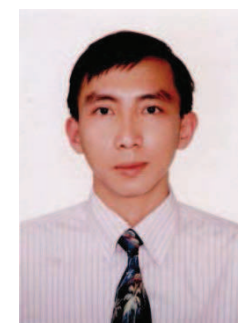

Vo Nguyen Quoc Bao was born in Khanh Hoa, Vietnam, in 1979. He received the B.E. and M.Eng. degree in electrical engineering from Ho Chi Minh City University of Technology (HCMUT), Vietnam, in 2002 and 2005, respectively, and Ph.D. degree in electrical engineering from University of U1san, South Korea, in 2010. In 2002, he joined the Department of Electrical Engineering, Posts and Telecommunications Institute of Technology (PTIT), as a lecturer. Since February 2010, he has been with the Department of Telecommunications, PTIT, where he is currently an Assistant Professor. His major research interests are modulation and coding techniques, MIMO system, combining technique, cooperative communications and cognitive radio. Dr. Bao is a Member of Korea Information and Communications Society (KICS), The Institute of Electronics, Information and Communication Engineers (IEICE) and the Institute of Electrical and Electronics Engineers (IEEE).

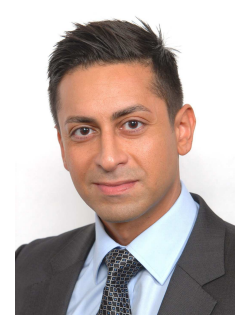

Maged Elkashlan (M'06) received the Ph.D. degree in Electrical Engineering from the University of British Columbia, Canada, 2006. From 2006 to 2007, he was with the Laboratory for Advanced Networking at University of British Columbia. From 2007 to 2011, he was with the Wireless and Networking Technologies Laboratory at Commonwealth Scientific and Industrial Research Organization (CSIRO), Australia. During this time, he held an adjunct appointment at University of Technology Sydney, Australia. In 2011, he joined the School of Electronic Engineering and Computer Science at Queen Mary University of London, UK, as an Assistant Professor. He also holds visiting faculty appointments at the University of New South Wales, Australia, and Beijing University of Posts and Telecommunications, China. His research interests fall into the broad areas of communication theory, wireless communications, and statistical signal processing for distributed data processing, millimeter wave communications, heterogeneous networks, and security.

Dr. Elkashlan currently serves as an Editor of IEEE TRANSACTIONS ON WiRELESS COMMUNICATIONS, IEEE TRANSACTIONS ON VEHICULAR TEChNology, and IEEE COMmunications LetTERs. He also serves as Lead Guest Editor for the special issue on "Green Media: The Future of Wireless Multimedia Networks" of the IEEE WIRELESS COMMUNICATIONS MAGAZINE, Lead Guest Editor for the special issue on "Millimeter Wave Communications for 5G" of the IEEE Communications MAGAZINE, Guest Editor for the special issue on "Energy Harvesting Communications" of the IEEE COMMUNICATIONS MAGAZINE, and Guest Editor for the special issue on "Location Awareness for Radios and Networks" of the IEEE JOURNAL ON Selected AReas in Communications. He received the Best Paper Award at the IEEE International Conference on Communications (ICC) in 2014, the International Conference on Communications and Networking in China (CHINACOM) in 2014, and the IEEE Vehicular Technology Conference (VTC-Spring) in 2013. He received the Exemplary Reviewer Certificate of the IEEE Communications Letters in 2012. 